Pacific

Journal of

Mathematics

\title{
GENERA AND FIBREDNESS OF MONTESINOS KNOTS
}

MiKami HiRASAWA AND Kunio MuRAsugi 


\title{
GENERA AND FIBREDNESS OF MONTESINOS KNOTS
}

\author{
Mikami Hirasawa AND Kunio Murasugi
}

\begin{abstract}
For Montesinos knots, we explicitly construct Seifert surfaces of minimal genus and solve the question of when they are fibred knots. For those of tunnel number one, we show that they are mostly fibred if their Alexander polynomials (of proper degrees) are monic.
\end{abstract}

\section{Introduction}

The class of Montesinos knots is one of many interesting special families of knots in $S^{3}$. As a generalization of 2-bridge knots, Montesinos knots have been studied for many years. In contrast to 2-bridge knots, some topological invariants of Montesinos knots cannot be determined from their algebraic invariants. For example, G. Burde [1984] partially determined the genus of a Montesinos knot and considered the question of when it is fibred, but since many Montesinos knots are homologically trivial, it is not easy to determine their genera. Burde calculated the Seifert matrix, and when it was not singular, he could determine the genus. However, he left many Montesinos knots untouched, because their matrices are singular.

In this paper, we completely determine the genus of all Montesinos knots, and solve the question of whether or not they are fibred. We use Gabai's geometric technique and avoid calculations of Seifert matrices, except in Section 8. Our results can be extended to Montesinos links, but for simplicity we consider only Montesinos knots, unless stated otherwise.

In Section 2, we recall some formulas on continued fractions, the notion of plumbing and deplumbing of an annulus, and theorems of Gabai [1986a] and Kanenobu [1979] on the incompressibility and fibredness conditions on pretzel links. In Section 3, we state our main theorems (Theorems 3.1 and 3.2), which are proved in the next four sections. In particular, in Section 6, we prove a key result (Theorem 6.2) which determines when a special Seifert surface is of minimal genus, and a

MSC2000: 57M25.

Keywords: Seifert surface, fibred knot, Montesinos knot, plumbing, rational tangle, continued

fraction, sutured manifold decomposition.

Hirasawa was partially supported by MEXT, Grant-in-Aid for Young Scientists (B) 14740048. Murasugi was partially supported by NSERC Grant A 4034. 
fibred surface. As an application of our main theorems, in Section 8 we study Montesinos knots of tunnel number one and show that most of them are fibred if their Alexander polynomials are monic. We provide examples at the end of each section.

\section{Preliminaries}

A Montesinos knot is a knot having a diagram of the form depicted in Figure 1, where $T\left(\frac{\beta_{i}}{\alpha_{i}}\right)$ (with $\alpha_{i}>1$ and $\operatorname{gcd}\left(\alpha_{i}, \beta_{i}\right)=1$ for each $i$ ) denotes a rational tangle of slope $\beta_{i} / \alpha_{i}$ and the twists on the right are opposite if $e$ is negative. We denote such a knot by

$$
K=M\left(\frac{\beta_{1}}{\alpha_{1}}, \frac{\beta_{2}}{\alpha_{2}}, \ldots, \frac{\beta_{r}}{\alpha_{r}} \mid e\right) .
$$

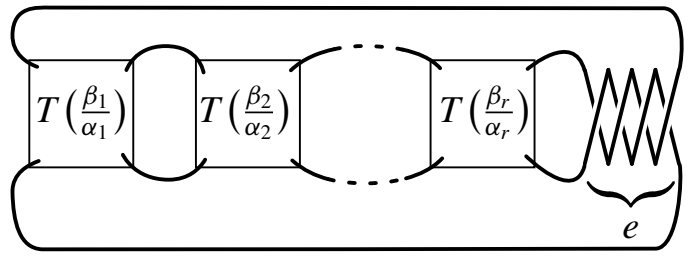

Figure 1. A Montesinos knot.

A specific example would be $M\left(\frac{2}{5}, \frac{1}{3},-\frac{2}{3} \mid-4\right)$ :

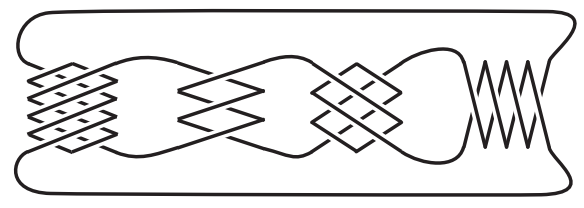

The upper left and lower left corners of $T(\beta / \alpha)$ are connected by a strand if and only if $\alpha$ is even. Hence we see that if $K=M\left(\frac{\beta_{1}}{\alpha_{1}}, \frac{\beta_{2}}{\alpha_{2}}, \ldots, \frac{\beta_{r}}{\alpha_{r}} \mid e\right)$ is to be a knot rather than a link, at most one of $\alpha_{1}, \alpha_{2}, \ldots, \alpha_{r}$ can be even. Since a cyclic permutation of indices does not change the knot type (see Remark 2.2), we hereafter assume that

$$
\alpha_{2}, \alpha_{3}, \ldots, \alpha_{r} \text { are odd. }
$$

With this convention, we say that $K$ is of odd type if $\alpha_{1}$ is odd, and of even type if $\alpha_{1}$ is even.

Diagrams. To find a suitable diagram of a Montesinos knot, we use continued fractions. A continued fraction expansion of a rational number $\beta / \alpha$, where we 
assume $-\alpha<\beta<\alpha$, is a finite sequence $c_{1}, c_{2}, \ldots, c_{m}$ such that

$$
\frac{\beta}{\alpha}=\frac{1}{c_{1}-\frac{1}{c_{2}-\frac{1}{\ddots \cdot-\frac{1}{c_{m}}}}}=:\left[c_{1}, c_{2}, \ldots, c_{m}\right]
$$

and $c_{1}, c_{2}, \ldots, c_{m} \neq 0$. The tangle $T(\beta / \alpha)$ is then representable as in Figure 2.
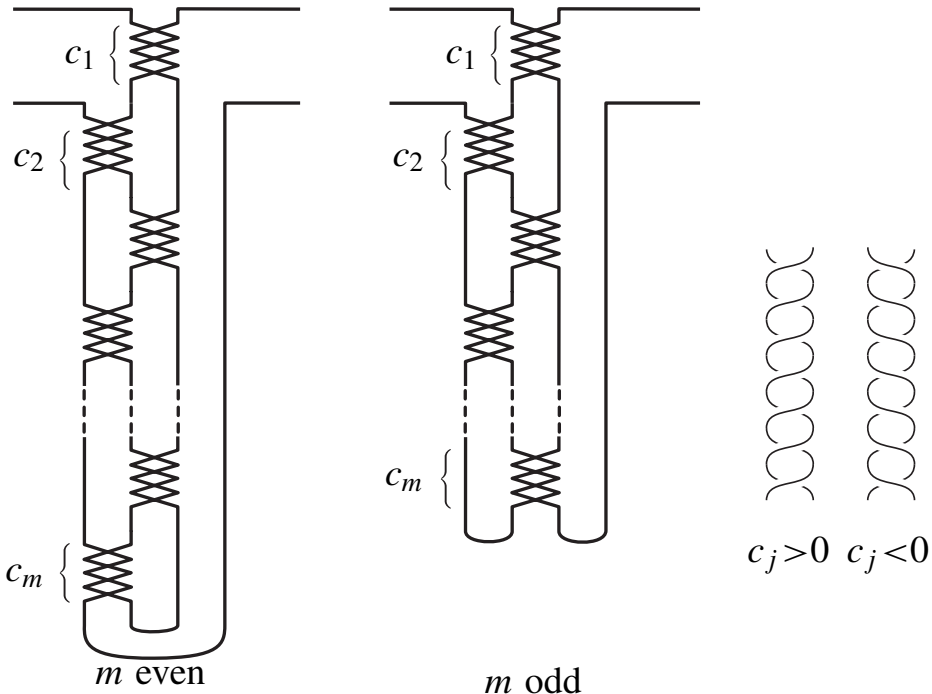

$m$ odd

Figure 2. Expansion of a rational tangle.

Using continued fractions, we obtain a new diagram of the Montesinos knot $K=M\left(\frac{\beta_{1}}{\alpha_{1}}, \frac{\beta_{2}}{\alpha_{2}}, \ldots, \frac{\beta_{r}}{\alpha_{r}} \mid e\right)$ as a composition of such diagrams plus $e$ twists, as depicted in Figure 3.

Examples 2.1. (1) $M\left(\frac{3}{7}, \frac{18}{43}, \frac{12}{41} \mid-4\right)$ is of odd type. Figure 11 depicts its diagram together with a Seifert surface of minimal genus.

(2) $M\left(\frac{3}{8}, \frac{3}{7}, \frac{4}{9} \mid 2\right)$ is of even type. Here, $\frac{3}{8}=[2,-2,-2], \frac{3}{7}=[2,-3]$ and $\frac{4}{9}=[2,-4]$.

Remark 2.2. A cyclic permutation of indices is induced by a flype (Figure 4), which does not change the knot type. This is not readily visible in Figure 3, but it follows from the symmetry of rational tangles. A diagram of the fraction $1+\beta / \alpha$ is interpreted as depicted in Figure 4, left. This is because we can calculate as follows: $1+\beta / \alpha=1 /(0-1 /(-1-\beta / \alpha)), 1+\beta / \alpha=1+\left[c_{1}, c_{2}, \ldots\right]=\left[0,-1, c_{1}, c_{2}, \ldots\right]$. Pictorially, if the first entry $c_{1}$ in Figure 2 is 0 , then the $c_{2}$ twistings can be merged 


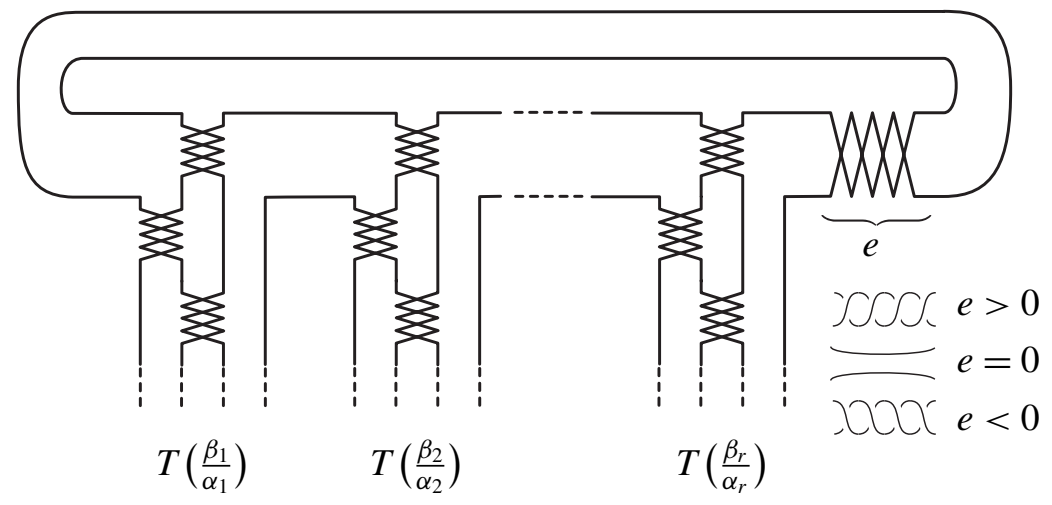

Figure 3. Another representation of a Montesinos knot.
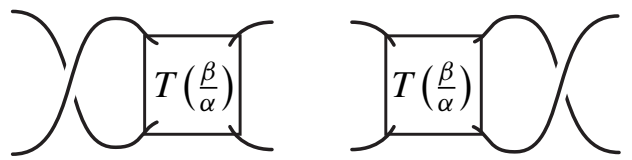

Figure 4. Sliding a crossing through a tangle.

with the $e$ twistings. Therefore, a fraction $\beta / \alpha$ can be freely replaced by $(\beta \pm \alpha) / \alpha$ at the expense of changing the value $e$ by $\mp 1$. A diagram of the fraction $-1+\beta / \alpha$ is similarly understood.

The following proposition is now immediate:

Proposition 2.3. The knots

$$
M\left(\frac{\beta_{1}}{\alpha_{1}}, \frac{\beta_{2}}{\alpha_{2}}, \ldots, \frac{\beta_{r}}{\alpha_{r}} \mid e\right) \quad \text { and } \quad M\left(\frac{\beta_{1}^{\prime}}{\alpha_{1}^{\prime}}, \frac{\beta_{2}^{\prime}}{\alpha_{2}^{\prime}}, \ldots, \frac{\beta_{r}^{\prime}}{\alpha_{r}^{\prime}} \mid e^{\prime}\right)
$$

are equivalent if, up to a cyclic permutations of indices, we have

$$
\frac{\beta_{i}}{\alpha_{i}} \equiv \frac{\beta_{i}^{\prime}}{\alpha_{i}^{\prime}}(\bmod 1) \text { for all } i \text { and } e+\sum_{i=1}^{r} \frac{\beta_{i}}{\alpha_{i}}=e^{\prime}+\sum_{i=1}^{r} \frac{\beta_{i}^{\prime}}{\alpha_{i}^{\prime}} .
$$

If $r \leq 2$, Montesinos knots are 2-bridge knots, for which our problems have been completely solved. Therefore, throughout this paper, we assume that

$$
3 \leq r \quad \text { and } \quad-\alpha_{i}<\beta_{i}<\alpha_{i} \text { for all } i .
$$

Example 2.4. $M\left(\frac{2}{3}, \frac{1}{5},-\frac{3}{5} \mid 3\right)$ and $M\left(-\frac{1}{3},-\frac{4}{5}, \frac{2}{5} \mid 4\right)$ are equivalent. 
Arithmetic of continued fractions. The continued fraction expansion of a rational number need not be unique. The following equalities hold, where on each line we choose top signs only or bottom signs only, and where we have used the obvious conventions $\left[c_{1}, \ldots, c_{j-1}, 0, c_{j+1}, \ldots, c_{m}\right]=\left[c_{1}, \ldots, c_{j-1}+c_{j+1}, \ldots, c_{m}\right]$ and $\left[c_{1}, \ldots, c_{j-1}, c_{j}, 0\right]=\left[c_{1}, \ldots, c_{j-1}\right]$ :

$$
\begin{aligned}
{\left[ \pm 2, c_{2}, \ldots\right] } & = \pm 1+\left[\mp 2, c_{2} \mp 1, \ldots\right] & & \\
{\left[\ldots, c_{i-1}, \pm 2, c_{i+1}, \ldots\right] } & =\left[\ldots, c_{i-1} \mp 1, \mp 2, c_{i+1} \mp 1, \ldots\right] & & \text { if } i \geq 2 \\
{\left[\ldots, c_{m-1}, \pm 2\right] } & =\left[\ldots, c_{m-1} \mp 1, \mp 2\right] & & \\
{\left[ \pm 1, c_{2}, \ldots\right] } & = \pm 1+\left[c_{2} \mp 1, \ldots\right], & & \\
{\left[\ldots, c_{i-1}, \pm 1, c_{i+1}, \ldots\right] } & =\left[\ldots, c_{i-1} \mp 1, c_{i+1} \mp 1, \ldots\right] & & \text { if } i \geq 2 \\
{\left[\ldots, c_{m-1}, \pm 1\right] } & =\left[\ldots, c_{m-1} \mp 1\right] & & \\
{\left[2,2, \ldots, 2, c_{k+1}, \ldots\right] } & =1+\left[-(k+1), c_{k+1}-1, \ldots\right] & & \text { if } k \geq 1 \\
{\left[-2, \ldots,-2, c_{k+1}, \ldots\right] } & =-1+\left[k+1, c_{k+1}+1, \ldots\right] & & \text { if } k \geq 1 .
\end{aligned}
$$

In particular,

$$
[\underbrace{2,2, \ldots, 2}_{k}]=1+[-(k+1)] \text { and }[\underbrace{-2,-2, \ldots,-2}_{k}]=-1+[k+1] \text {. }
$$

The "top" equalities in (1) and (4) (involving the positive sign on the left-hand side) can be checked by reference to Figure 5 and Figure 6, respectively; the figures show the case that $m$ is odd, but that assumption is not essential. To obtain the "bottom" equalities in (1) and (4), take mirror images. We omit pictorial proofs for the other equalities, but they follow directly from calculations on continued fractions. To prove (7), first apply (1), then apply (5) repeatedly.

Strict continued fractions. Let $S=\left[x_{1}, x_{2}, \ldots, x_{m}\right]$ be a continued fraction. We call $S$ an even continued fraction if all the $x_{j}$ are even. We call $S$ a strict continued fraction if

(a) $x_{j}$ is even for any odd $j$, and

(b) $x_{j} x_{j+1}<0$ whenever $j$ is odd and $\left|x_{j}\right|=2$.

Proposition 2.5. Suppose that $\alpha$ is odd, $-\alpha<\beta<\alpha$ and $\alpha>2|\beta|$. Then $\frac{\beta}{\alpha}$ has a strict continued fraction.

Note that by replacing $\beta / \alpha$ by $\pm 1+(\beta \mp \alpha) / \alpha$, we may assume $\alpha>2|\beta|$.

Proof. (1) If $\beta$ is even, $\frac{\beta}{\alpha}$ has a (unique) even continued fraction $\left[2 c_{1}, 2 c_{2}, \ldots, 2 c_{m}\right]$ of even length. Since $\alpha>2|\beta|$, it follows that $\left|c_{1}\right| \neq 1$ or $c_{1} c_{2}<0$. Therefore, we ignore $2 c_{1}$ and proceed to $2 c_{3}$. Apply (2) or (3) repeatedly, if necessary, to obtain the strict form. 


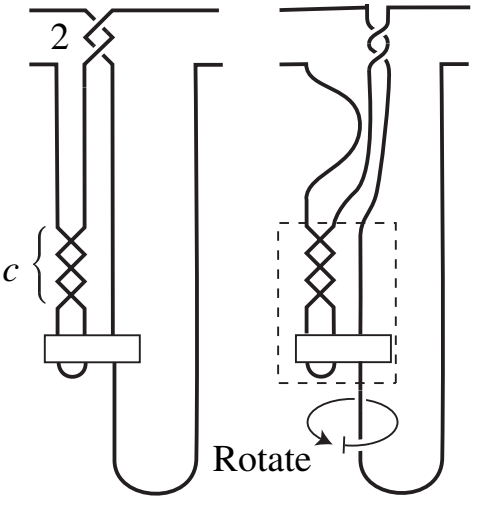

(a)

(b)

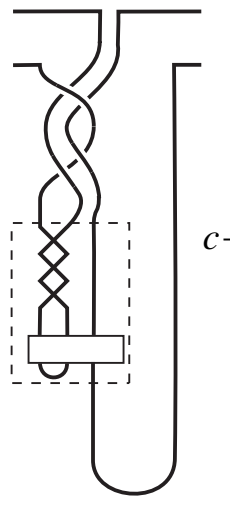

(c)

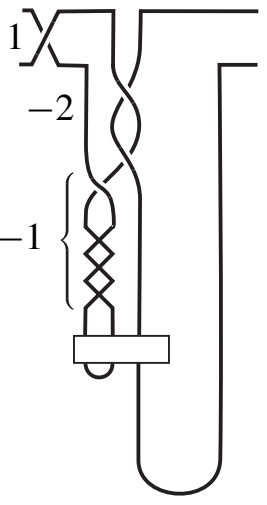

(d)

Figure 5. Pulling out a twist, I.

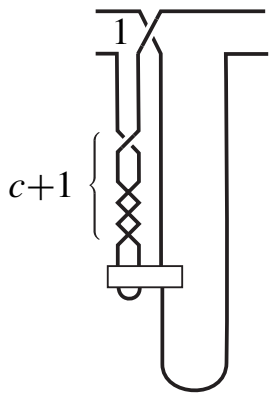

(a)

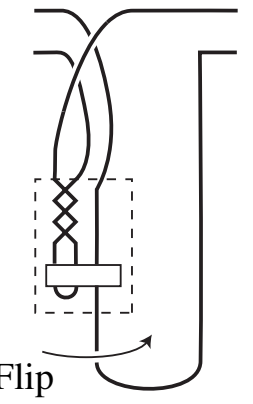

(b)

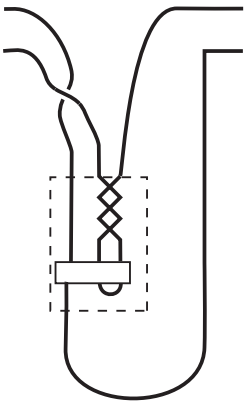

(c)

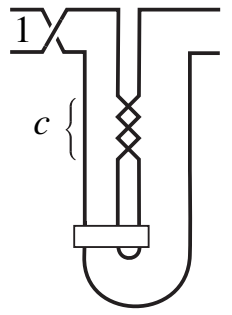

$(d)$

Figure 6. Pulling out a twist, II.

(2) If $\beta$ is odd, $\frac{\beta}{\alpha}$ has a unique "almost even" continued fraction

$$
S=\left[2 c_{1}, 2 c_{2}, \ldots, 2 c_{m-1}, c_{m}\right],
$$

where $c_{m}$ is odd, $2 a+1$ say, and $|2 a+1| \geq 3$. If $m$ is odd, apply (6) and replace $S$ by $S^{\prime}=\left[2 c_{1}, 2 c_{2}, \ldots, 2 c_{m-1}, 2 a,-1\right]$ of even length. Apply the previous argument to obtain the strict form.

Examples 2.6. (1) The continued fractions $\frac{6}{23}=[4,6]$ and $\frac{8}{19}=[2,-3,-2,1]$ are strict.

(2) $\frac{7}{10}=[2,2,4]$ is not strict, but $\frac{-3}{10}=[-4,-2,-2]$ is strict. We can also calculate $\frac{7}{10}=[2,2,4]=1+[-2,1,4]$, and hence $\frac{-3}{10}=[-2,1,4]$. Note that $[-4,-2,-2]$ and $[-2,1,4]$ are both strict continued fractions of $-\frac{3}{10}$. These are related by Equations (1)-(6). 
Pretzel links. Recall that a pretzel link is one of the form shown in Figure 1, with each $\beta_{i}$ equal to 1 .

Theorem 2.7 [Gabai 1986b]. Let $K=P\left(n_{1}, n_{2}, \ldots, n_{r}\right)$ be an (oriented) pretzel link, where the $n_{i}$, for $1 \leq i \leq r$, are nonzero and either all odd or all even. Assume that $K$ spans a Seifert surface $F$ consisting of two disks to which bands $B_{i}$ $(1 \leq i \leq r)$ with $n_{i}$ twists are attached. (Such a pretzel link is said to be natural.) Then $F$ is a Seifert surface of minimal genus if and only if either all the $n_{i}$ are even and $\left(n_{1}, n_{2}, \ldots, n_{r}\right) \neq \pm(2,-2, \ldots, 2,-2)$, or all the $n_{i}$ are odd and all the $n_{j}$ with $\left|n_{j}\right|=1$ are of the same sign.

For the fibredness of $F$, we will use the following theorem of T. Kanenobu [1979] (see also [Gabai 1986a]).

Theorem 2.8. Let $K$ and $F$ be as in Theorem 2.7.

(1) Suppose the $n_{i}$ are all odd. $F$ is a fibre surface if and only if $\left(n_{1}, n_{2}, \ldots, n_{r}\right)$ is a permutation of $\pm(\underbrace{3,3, \ldots, 3}_{a}, \underbrace{-1,-1, \ldots,-1}_{b})$, where $a \geq 0$ and $b>0$.

(2) Suppose the $n_{i}$ are all even. $F$ is a fibre surface if and only if $\left(n_{1}, n_{2}, \ldots, n_{r}\right)$ is a cyclic permutation of

$$
\pm(2,-2, \ldots, 2,-2,2,-4) \text { or } \pm(2,-2, \ldots, 2,-2,2 q),
$$

where $q$ is an integer.

Deplumbing of annuli. Plumbing (see Figure 7) is a way to glue two Seifert surfaces to obtain a new surface. Here is a special case of Gabai's theorem for the additivity of genera and fibredness under the Murasugi sum:

Theorem 2.9 [Gabai 1983; 1985]. Suppose that a Seifert surface $F$ is a plumbing of $F_{0}$ and a nontrivial annulus $A$. Then $F$ is a minimal genus Seifert surface for $\partial F$ if and only if $F_{0}$ is a minimal genus Seifert surface for $\partial F_{0}$. Furthermore, $F$ is a fibre surface if and only if $F_{0}$ is a fibre surface and the annulus $A$ is a Hopf band (an unknotted annulus with a full-twist).

Lemma 2.10 (Deplumbing lemma). Suppose that a Seifert surface $F$ is obtained by attaching a band $B$ to a Seifert surface $F_{0}$. Let $c$ be the core of $B$ such that $c \cap F_{0}=\partial c$. Suppose further, there is an arc d properly embedded in $F_{0}$ such that $c \cap d=\partial c=\partial d$. If the push off of the loop $c \cup d$ can be separated from $F_{0}$ by a sphere, then cutting the band $B$ in $F$ is equivalent to deplumbing an annulus $A$ from $F$, where $A$ is the union of the band $B$ and a neighbourhood of $d$ in $F_{0}$. (See Figure 7.)

The proof is easy and we omit it.

Let $A(c)$ denote a $c$ half-twisted annulus with a trivial core. (See Figure 8.) 


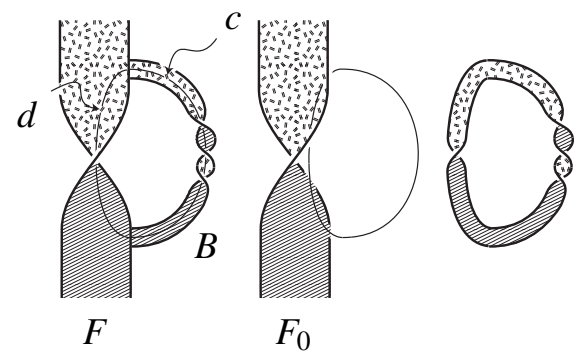

Figure 7. Deplumbing an annulus.

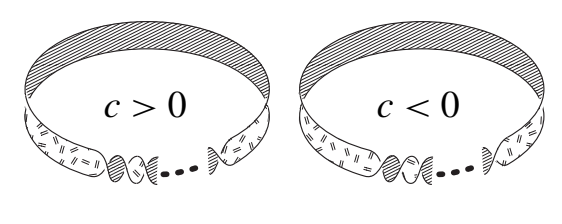

Figure 8. Convention for annuli twists.

If $|c|=2$, then $A(c)$ is a Hopf band and is sometimes denoted by $H(c)$. From now on, let $\varepsilon(c)$ denote $c /|c|$, where $c$ is a nonzero integer.

Example 2.11. Figure 9 depicts "obvious" deplumbings of annuli, where the loops represent the cores of annuli to be deplumbed. The surfaces depend on the local orientations of the link.

(1) Consider the (partial) Seifert surface $F$ depicted in Figure 9, left, corresponding to the continued fraction $[\ldots, p, 2 q, s]$. The process

$$
[\ldots, p, 2 q, s] \rightarrow[\ldots, p, 2 q]
$$

is realized by deplumbing $|s|-1$ Hopf bands $H(-2 \varepsilon(s))$. The process

$$
[\ldots, p, 2 q] \rightarrow[\ldots, p]
$$

is realized by deplumbing an annulus $A(2 q-\varepsilon(s)-\varepsilon(p))$.

(2) In Figure 9, right, the process $[\ldots, 2 p, 2 q, 2 s] \rightarrow[\ldots, 2 p, 2 q] \rightarrow[\ldots, 2 p]$ is realized by first deplumbing an annulus $A(2 s)$, and then an annulus $A(2 q)$.
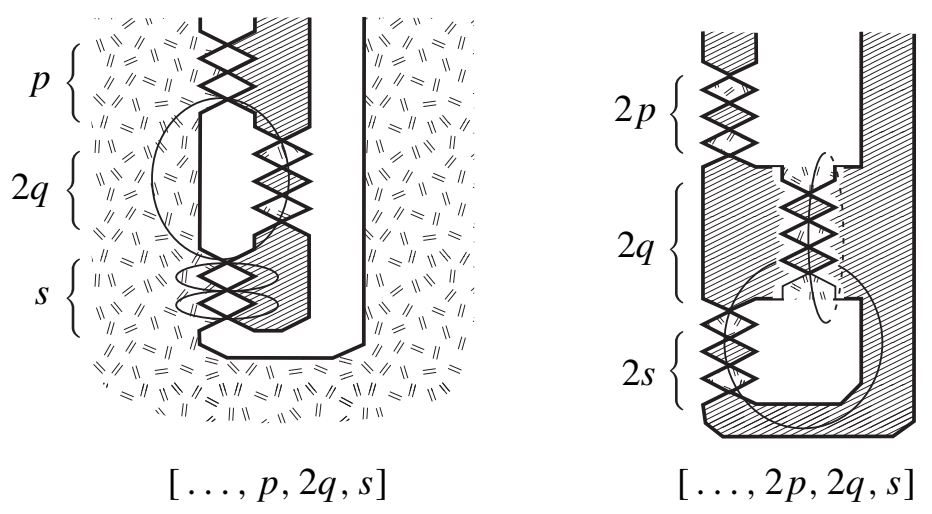

Figure 9. Examples of deplumbing. 
Note that in (1), $A(2 q-\varepsilon(s)-\varepsilon(p))$ is compressible if $2 q-\varepsilon(s)-\varepsilon(p)=0$. One of the reasons to introduce strict continued fractions is to avoid that situation. Another will be found in the proof of Theorem 3.1.

To calculate the genus $g(F)$ of a Seifert surface $F$, it is sometimes convenient to use the Betti number $\beta(F)$, i.e., the rank of the integral homology group $H_{1}(F ; \mathbb{Z})$. We have

$$
g(F)=\frac{1}{2} \beta(F)-\mu+1,
$$

where $\mu$ denotes the number of connected components of $\partial F$.

Proposition 2.12. If $F$ is obtained from $F_{0}$ by successive applications of plumbing $k$ annuli, then $\beta(F)=\beta\left(F_{0}\right)+k$.

\section{Main Theorems}

Let $K=M\left(\frac{\beta_{1}}{\alpha_{1}}, \frac{\beta_{2}}{\alpha_{2}}, \ldots, \frac{\beta_{r}}{\alpha_{r}} \mid e\right)$ be a Montesinos knot. Assume that $r \geq 3$ and that, for all $i=1,2, \ldots, r$, we have $\alpha_{i}>1$ and $-\alpha_{i}<\beta_{i}<\alpha_{i}$, with $\operatorname{gcd}\left(\alpha_{i}, \beta_{i}\right)=1$.

First we consider Montesinos knots of odd type.

Theorem 3.1. Suppose that all $\alpha_{i}$ are odd, and further that $2\left|\beta_{i}\right|<\alpha_{i}$ (by replacing $\beta_{i} / \alpha_{i}$ by $\pm 1+\beta_{i} / \alpha_{i}$, if necessary). For each $i$, let $S_{i}=\left[2 a_{1}{ }^{(i)}, b_{1}{ }^{(i)}, 2 a_{2}{ }^{(i)}, b_{2}{ }^{(i)}\right.$, $\left.\ldots, 2 a_{q_{i}}{ }^{(i)}, b_{q_{i}}{ }^{(i)}\right]$ be a strict continued fraction of $\beta_{i} / \alpha_{i}$.

(I) $g(K)=\frac{1}{2}\left(\sum_{i=1}^{r} b^{(i)}+|e|-1\right)$, where $b^{(i)}=\sum_{j=1}^{q_{i}}\left|b_{j}{ }^{(i)}\right|$.

(II) (a) If $e=0, K$ is never fibred.

(b) Suppose $e \neq 0$. Then $K$ is a fibred knot if and only if the following three conditions are satisfied.

(1) $\left|a_{j}{ }^{(i)}\right|=1$ or 2 , for any $i, j, 1 \leq i \leq r, 1 \leq j \leq q_{i}$.

(2) (i) If $\left|a_{1}{ }^{(i)}\right|=1$, then $a_{1}^{(i)} e<0$.

(ii) If $\left|a_{1}{ }^{(i)}\right|=2$, then $a_{1}{ }^{(i)} b_{1}{ }^{(i)}>0$ and $a_{1}{ }^{(i)} e<0$.

(3) For any $j>1$,

(i) if $\left|a_{j}{ }^{(i)}\right|=1$, then $b_{j-1}{ }^{(i)} b_{j}{ }^{(i)}<0$;

(ii) if $\left|a_{j}{ }^{(i)}\right|=2$, then $a_{j}{ }^{(i)} b_{j-1}^{(i)}>0$ and $a_{j}{ }^{(i)} b_{j}{ }^{(i)}>0$.

(Note that $a_{j}{ }^{(i)} b_{j}{ }^{(i)}<0$ in (3i), since $S_{i}$ is strict.)

See Figure 11 on page 64 for an example of a minimal genus Seifert surface for a knot of odd type.

Next we consider Montesinos knots of even type.

Theorem 3.2. Suppose that $\alpha_{1}$ is even and that for any $i \geq 2, \alpha_{i}$ is odd and $\beta_{i}$ is even. Assume e to be even (by replacing $\beta_{1} / \alpha_{1}$ by $\pm 1+\beta_{1} / \alpha_{1}$, if necessary). Let $S_{i}=\left[2 c_{1}{ }^{(i)}, 2 c_{2}{ }^{(i)}, 2 c_{3}{ }^{(i)}, \ldots, 2 c_{m_{i}}{ }^{(i)}\right]$ be the even continued fraction of $\beta_{i} / \alpha_{i}$, for $i=1,2, \ldots, r$. Note that $m_{1}$ is odd and $m_{i}$ is even for $i>1$. 
(I) Suppose $e \neq 0$. Then $g(K)=\frac{1}{2}\left(1+\sum_{i=1}^{r} m_{i}\right)$, and $K$ is a fibred knot if and only if $|e|=2$ and $\left|c_{j}{ }^{(i)}\right|=1$ for any $i$ and $j$; equivalently, if and only if $e= \pm 2$ and all 2-bridge knots and a 2-bridge link $B\left(\beta_{i} / \alpha_{i}\right)$ are fibred.

(II) Suppose $e=0$ and $\left(c_{1}{ }^{(1)}, c_{1}{ }^{(2)}, \ldots, c_{1}{ }^{(r)}\right) \neq \pm(1,-1, \ldots, 1,-1)$. Then $g(K)=\frac{1}{2}\left(-1+\sum_{i=1}^{r} m_{i}\right)$, and $K$ is fibred if and only if the following two conditions are satisfied:

(i) $\left(c_{1}{ }^{(1)}, c_{1}{ }^{(2)}, \ldots, c_{1}{ }^{(r)}\right)$ is a cyclic permutation of

$$
\pm(1,-1, \ldots, 1,-1,1,-2) \quad \text { or of } \quad \pm(1,-1, \ldots, 1,-1, n), n \in \mathbb{Z} \text {. }
$$

(ii) $\left|c_{j}{ }^{(i)}\right|=1$ for any $i, 1 \leq i \leq r$ and $j, 2 \leq j \leq m_{i}$.

(III) Suppose $e=0$ and $\left(c_{1}{ }^{(1)}, c_{1}{ }^{(2)}, \ldots, c_{1}{ }^{(r)}\right)= \pm(1,-1, \ldots, 1,-1)$. By taking the mirror image, if necessary, assume that

$$
\left(c_{1}^{(1)}, c_{1}{ }^{(2)}, \ldots, c_{1}^{(r)}\right)=(1,-1, \ldots, 1,-1) .
$$

Define $p_{i}$ as the number of leading 2's in $S_{i}$, if $i$ is odd, or the number of leading -2 's in $S_{i}$, if $i$ is even. Let $p=\min \left\{p_{1}, \ldots, p_{r}\right\}$. Then $g(K)=$ $\frac{1}{2}\left(1+\sum_{i=1}^{r} m_{i}\right)-(p+1)$, and $K$ is a fibred knot if and only if the following conditions are satisfied:

(i) for $i \geq 1,\left|c_{j}^{(i)}\right|=1$, for $j=p+2, p+3, \ldots, m_{i}$;

(ii) among the pairs $\left(2 c_{p+1}{ }^{(1)}-1,2 c_{p+1}{ }^{(2)}+1\right),\left(2 c_{p+1}{ }^{(3)}-1,2 c_{p+1}{ }^{(4)}+1\right)$, $\ldots,\left(2 c_{p+1}{ }^{(r-1)}-1,2 c_{p+1}{ }^{(r)}+1\right)$, all except one are the pair $(1,-1)$, and the exceptional pair is one of the following: $(1, \phi),(\phi,-1),(3,-1)$ or $(1,-3)$, where, if the length of $S_{i}$ is $p$, then $2 c_{p+1}^{(i)}-1$ is denoted by $\phi$. (For the definition of $\phi$, see Section 6.)

See Figures 12 and 13 for minimal genus Seifert surfaces for cases (I) and (II) respectively. For case (III), we need more complicated Seifert surfaces constructed from those in Section 6.

\section{Proof of Theorem 3.1}

Let $K=M\left(\frac{\beta_{1}}{\alpha_{1}}, \frac{\beta_{2}}{\alpha_{2}}, \ldots, \frac{\beta_{r}}{\alpha_{r}} \mid e\right)$ be a Montesinos knot of odd type. By Proposition 2.5 , each of $\beta_{i} / \alpha_{i}$ has a strict continued fraction,

$$
\left[2 a_{1}{ }^{(i)}, b_{1}{ }^{(i)}, 2 a_{2}{ }^{(i)}, b_{2}{ }^{(i)}, \ldots, 2 a_{q_{i}}{ }^{(i)}, b_{q_{i}}{ }^{(i)}\right] .
$$

Let $D$ be the diagram of $K$ obtained from these continued fractions:

First we note that $K$ is a knot if and only if $\sum_{i=1}^{r} b^{(i)}+e \equiv 1(\bmod 2)$, where $b^{(i)}=\sum_{j=1}^{q_{i}}\left|b_{j}{ }^{(i)}\right|$. We apply the Seifert algorithm on $D$ and obtain a Seifert surface $F$ depicted in Figure 10.

Proposition 4.1. The Betti number of $F$ is given by $\beta(F)=\sum_{i=1}^{r} b^{(i)}+|e|-1$. 


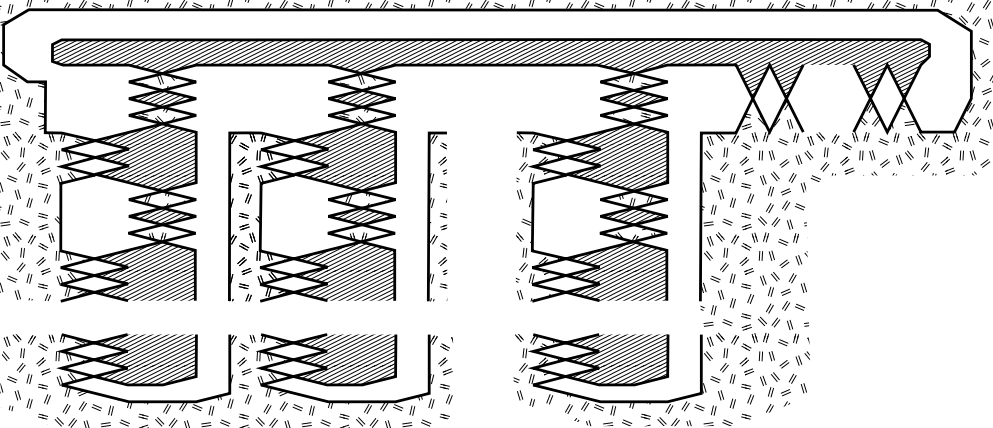

Figure 10. A minimal Seifert surface for knots of odd type.

Proof. Applying Example 2.11(1) to each 2-tangle $T\left(\beta_{i} / \alpha_{i}\right)$, we can deplumb $\sum_{j=1}^{q_{i}}\left(\left|b_{j}{ }^{(i)}\right|-1\right)$ Hopf bands and $q_{i}-1$ annuli $A\left(2 a_{j}{ }^{(i)}-\varepsilon\left(b_{j-1}{ }^{(i)}\right)-\varepsilon\left(b_{j}{ }^{(i)}\right)\right)$. Since each continued fraction is strict, $\left|2 a_{j}{ }^{(i)}-\varepsilon\left(b_{j-1}{ }^{(i)}\right)-\varepsilon\left(b_{j}{ }^{(i)}\right)\right| \geq 2$. Therefore the total number of (nontrivial) annuli (including Hopf bands) to be deplumbed is $\sum_{i=1}^{r} \sum_{j=1}^{q_{i}}\left(\left|b_{j}{ }^{(i)}\right|-1\right)+\sum_{i=1}^{r}\left(q_{i}-1\right)=\sum_{i=1}^{r} \sum_{j=1}^{q_{i}}\left|b_{j}{ }^{(i)}\right|-r=\sum_{i=1}^{r} b^{(i)}-r$.

Let $F_{0}$ be the surface obtained from $F$ by deplumbing these annuli. Then $\partial F_{0}$ is the natural pretzel link

$$
P\left(2 a_{1}{ }^{(1)}-\varepsilon\left(b_{1}{ }^{(1)}\right), 2 a_{1}{ }^{(2)}-\varepsilon\left(b_{1}{ }^{(2)}\right), \ldots, 2 a_{1}{ }^{(r)}-\varepsilon\left(b_{1}{ }^{(r)}\right), \varepsilon(e), \ldots, \varepsilon(e)\right),
$$

and hence, $\beta\left(F_{0}\right)=r+|e|-1$. Therefore,

$$
\beta(F)=\beta\left(F_{0}\right)+\sum_{i=1}^{r} b^{(i)}-r=\sum_{i=1}^{r} b^{(i)}+|e|-1 .
$$

Now, $F$ is a minimal genus Seifert surface for $K$. In fact, by strictness of continued fractions, $\left|2 a_{1}{ }^{(i)}-\varepsilon\left(b_{1}{ }^{(i)}\right)\right| \geq 3$, and hence, by Theorem 2.7, $F_{0}$ is a minimal genus Seifert surface for $\partial F_{0}$. Therefore, $F$ is of minimal genus. This proves Theorem 3.1(I).

Next, suppose that $F$ is a fibre surface. Then $F_{0}$ must be a fibre surface and every annulus deplumbed from $F$ is a Hopf band. However, if $e=0$, then $\partial F_{0}$ is the natural pretzel link $P\left(2 a_{1}{ }^{(1)}-\varepsilon\left(b_{1}{ }^{(1)}\right), 2 a_{1}{ }^{(2)}-\varepsilon\left(b_{1}{ }^{(2)}\right), \ldots, 2 a_{1}{ }^{(r)}-\varepsilon\left(b_{1}{ }^{(r)}\right)\right)$. Since $\left|2 a_{1}{ }^{(i)}-\varepsilon\left(b_{1}{ }^{(i)}\right)\right| \geq 3$, for any $i, F_{0}$ is not a fibre surface by Theorem 2.8(1). Therefore, $K$ is not fibred, which proves Theorem 3.1(IIa).

Suppose $e \neq 0$. Then the condition that $F_{0}$ be a fibre surface implies that for any $i$,

$$
\left|2 a_{1}{ }^{(i)}-\varepsilon\left(b_{1}{ }^{(i)}\right)\right|=3 \quad \text { and } \quad e\left(2 a_{1}{ }^{(i)}-\varepsilon\left(b_{1}{ }^{(i)}\right)\right)<0 .
$$


The first condition is equivalent to

$$
\left|a_{1}{ }^{(i)}\right|=1 \text { or } 2 \quad \text { and } \quad \text { if }\left|a_{1}{ }^{(i)}\right|=2 \text {, then } 2 a_{1}{ }^{(i)} b_{1}{ }^{(i)}>0 .
$$

Since $2 a_{1}{ }^{(i)}-\varepsilon\left(b_{1}{ }^{(i)}\right)$ and $2 a_{1}{ }^{(i)}$ have the same sign, the second condition in (9) is equivalent to $a_{1}{ }^{(i)} e<0$. Further, if $\left|a_{1}{ }^{(i)}\right|=1$, then $a_{1}{ }^{(i)} b_{1}{ }^{(i)}<0$ by the strictness assumption and $a_{1}{ }^{(i)} e<0$, and hence, condition (b2) is satisfied. Finally, the same argument shows that the condition that every annulus deplumbed from $F$ be a Hopf band implies condition (b3) in Theorem 3.1.

The converse is obvious. The proof of Theorem 3.1 is complete.

Example 4.2. Let $K=M\left(-\frac{4}{7}, \frac{18}{43}, \frac{12}{41} \mid-3\right)$. Since $2|-4|>7$, we replace $K$ by $K^{\prime}=M\left(\frac{3}{7}, \frac{18}{43}, \frac{12}{41} \mid-4\right)$. A Seifert surface $F$ for $K^{\prime}$ is depicted in Figure 11. The Betti number of $F$ is 16 and hence, $g(F)=8$. Furthermore, $F$ is a fibre surface by Theorem 3.1. We should note that none of the 2-bridge knots $B\left(\frac{3}{7}\right), B\left(\frac{18}{43}\right)$ and $B\left(\frac{12}{41}\right)$ is a fibred knot.

\section{Proof of Theorem 3.2(I)}

In this section, we prove parts (I) and (II) of Theorem 3.2. The last part (III) will be proved in Section 7.

Let $K=M\left(\frac{\beta_{1}}{\alpha_{1}}, \frac{\beta_{2}}{\alpha_{2}}, \ldots, \frac{\beta_{r}}{\alpha_{r}} \mid e\right)$ be a Montesinos knot of even type. We express each fraction $\beta_{i} / \alpha_{i}$ as an even continued fraction, and write, for $i \geq 1$,

$$
\frac{\beta_{i}}{\alpha_{i}}=\left[2 c_{1}{ }^{(i)}, 2 c_{2}{ }^{(i)}, 2 c_{3}{ }^{(i)}, \ldots, 2 c_{m_{i}}{ }^{(i)}\right] .
$$

Note that $m_{1}$ is odd and $m_{i}$ is even for $i>1$.

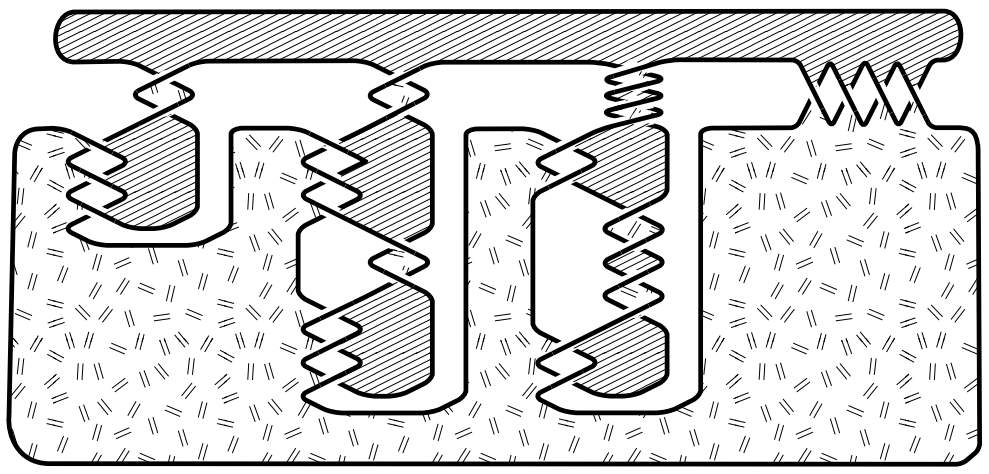

Figure 11. $M\left(\frac{3}{7}, \frac{18}{43}, \frac{12}{41} \mid-4\right) .\left(\frac{3}{7}=[2,-3], \frac{18}{43}=[2,-3,-2,3]\right.$, $\frac{12}{41}=[4,2,4,2]$.) 
Case 1: $e \neq 0$. Using (10), we construct a diagram $D$ of $K$ as in Figure 12, and apply the Seifert algorithm to obtain a Seifert surface $F$ for $K$.

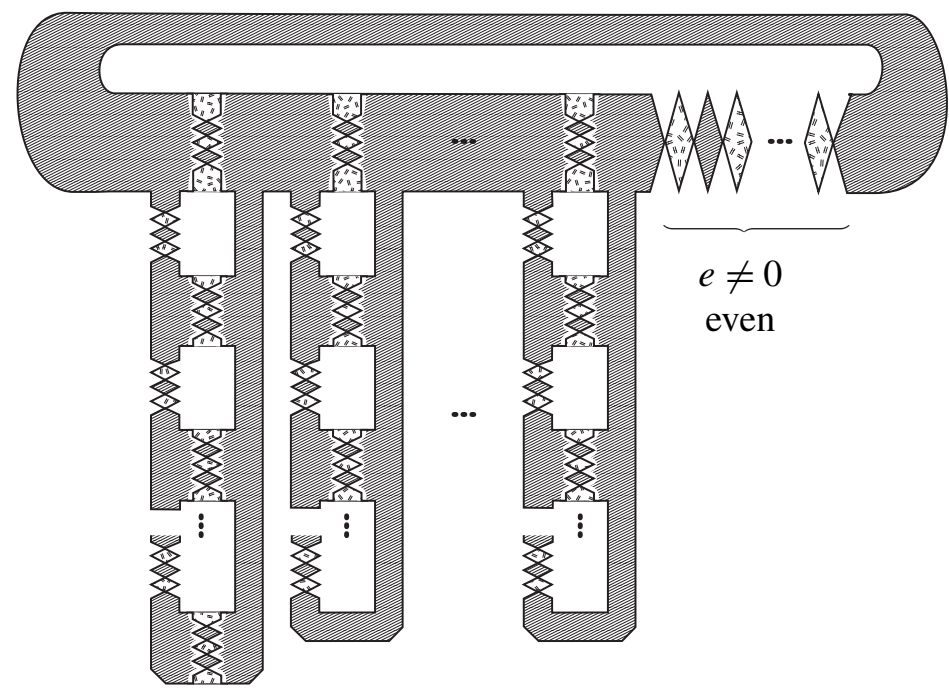

Figure 12. A minimal Seifert surface for knots of even type, $e \neq 0$.

Using Example 2.11(2) on each tangle $T\left(\frac{\beta_{i}}{\alpha_{i}}\right)$ (see Figure 9), we can deplumb $\sum_{i=1}^{r} m_{i}$ annuli $A\left(2 c_{j}{ }^{(i)}\right), 1 \leq i \leq r, 1 \leq j \leq m_{i}$. Since $\beta(A(e))=1$, we have

$$
\beta(F)=1+\sum_{i=1}^{r} m_{i} .
$$

Since $A(e)$ is of minimal genus, $F$ is a minimal genus Seifert surface for $K$ of genus $\frac{1}{2}\left(1+\sum_{i=1}^{r} m_{i}\right)$.

If $F$ is a fibre surface, we see immediately that $\left|c_{j}{ }^{(i)}\right|=1$ for all $i$ and $j$, and $|e|=2$, and vice versa. This proves (I). The last statement follows from the fact that a 2-bridge knot or link is fibred if and only if each entry of the even continued fraction is \pm 2 .

Case 2: $e=0$ and $\left(c_{1}{ }^{(1)}, c_{1}{ }^{(2)}, \ldots, c_{1}{ }^{(r)}\right) \neq \pm(1,-1, \ldots, 1,-1)$. We use the same surface $F$ depicted in Figure 12. However, since $e$ vanishes, $F$ is compressible, and the compression yields the surface $\widetilde{F}$ depicted in Figure 13.

As we did in case (I), deplumb all annuli $A\left(2 c_{j}^{(i)}\right), 1 \leq i \leq r, j \geq 2$ from $\widetilde{F}$. Then we have the natural pretzel link $P\left(2 c_{1}{ }^{(1)}, 2 c_{1}{ }^{(2)}, \ldots, 2 c_{1}{ }^{(r)}\right)$. Since $\left(c_{1}{ }^{(1)}, c_{1}{ }^{(2)}, \ldots, c_{1}{ }^{(r)}\right) \neq \pm(1,-1, \ldots, 1,-1)$, it follows from Theorem 2.7 that $F_{0}$, the spanning surface for this natural pretzel link, is of minimal genus, and hence so is $\widetilde{F}$. The genus of $\widetilde{F}$ is

$$
g(\widetilde{F})=\frac{1}{2} \beta(\widetilde{F})=\frac{1}{2}\left(\sum_{i=1}^{r} m_{i}-1\right) .
$$




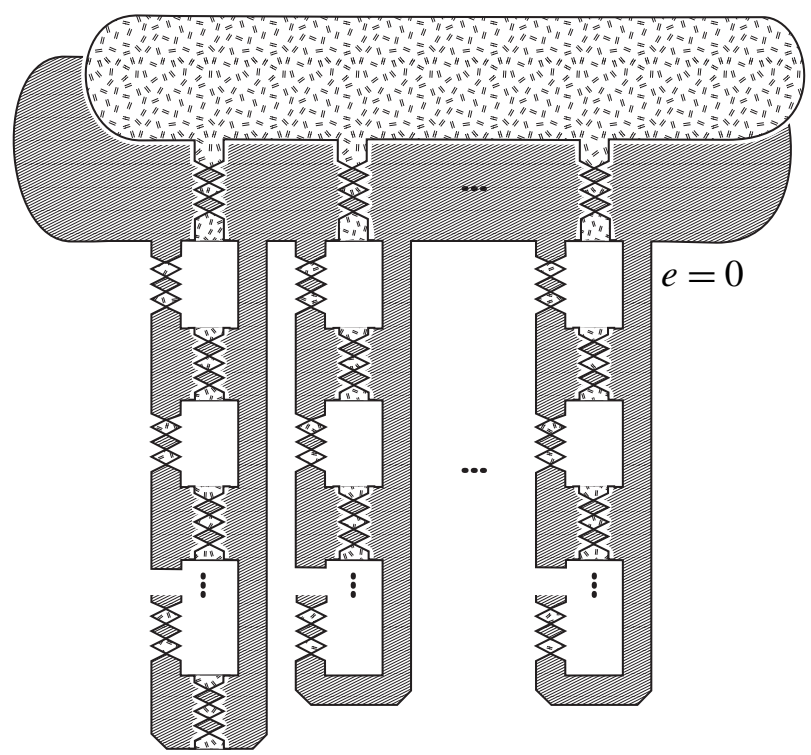

Figure 13. A minimal Seifert surface for knots of even type with $e=0$ (general case).

Furthermore, it follows from Theorem 2.8(2) that the conditions for $\widetilde{F}$ to be fibred are given by (II).

This concludes the proof of Theorem 3.2, parts (I) and (II).

Examples 5.1. (1) Let $K=M\left(\frac{7}{10}, \frac{2}{9}, \frac{4}{7} \mid 2\right)$. Since $\frac{7}{10}=[2,2,4], \frac{2}{9}=[4,-2]$ and $\frac{4}{7}=[2,4]$, we see from Theorem $3.2(\mathrm{I}), g(K)=4$, but some entry of a continued fraction is not \pm 2 , and thus $K$ is not fibred.

(2) Let $K=M\left(\frac{5}{8},-\frac{2}{3}, \frac{2}{7} \mid 0\right)$. Since $\frac{5}{8}=[2,2,-2],-\frac{2}{3}=[-2,-2]$ and $\frac{2}{7}=[4,2]$, by Theorem 3.2(II), we see $g(K)=3$ and $K$ is a fibred knot, although $B\left(\frac{2}{7}\right)$ is not fibred.

\section{A special Seifert surface}

To construct Seifert surfaces of minimal genus for the knots left out in the previous section and to prove Theorem 3.2(III), we need a new Seifert surface that may not be obtained by the Seifert algorithm. Similar surfaces were studied in [Gabai 1986a].

Let $F(n, m)$ be a Seifert surface depicted in Figure 14, which consists of (i) two disks $D_{1}$ and $D_{2}$, (ii) $m(n-2)$ bands $B_{i}^{(j)}, 1 \leq i \leq m, 1 \leq j \leq n-2$, and (iii) $n$ sheets of $m$-punctured spheres $\Sigma_{1}, \ldots, \Sigma_{n}$, where each $\Sigma_{j}$ is bounded by $m$ circles $C_{1}^{(j)}, \ldots, C_{m}^{(j)}$. 
If $n=1$, then $F(1, m)$ consists of a pair of $m$ punctured spheres. We omit this case since the arguments are similar.

In Figure 14, we immediately see that $F(n, m)$ is a Seifert surface for the natural pretzel link $P(-(n+1), n+1,-(n+1), n+1, \ldots,-(n+1), n+1)$.

Now we attach to $F(n, m)$ at most $2 m$ mutually disjoint bands $X_{1}, Y_{1}, \ldots, X_{m}, Y_{m}$, as in Figure 15. Bands $X_{i}$ and $Y_{i}$ have an odd number of twists, say $a_{i}$ and $b_{i}$, respectively. The signs of $a_{i}$ and $b_{i}$ are defined in Figure 15.

The surface $F(n, m)$ with bands $X_{i}, Y_{j}$ attached is denoted by $F=F(n, m \mid$ $\left.\left(a_{1}, b_{1}\right),\left(a_{2}, b_{2}\right), \ldots,\left(a_{m}, b_{m}\right)\right)$. If $X_{i}$ (or $\left.Y_{i}\right)$ does not exist, we replace $a_{i}$ (or $b_{i}$ ) by a symbol $\phi$. Note that $F$ is a connected surface if and only if at least one band is attached. We assume for any $i, a_{i} \neq-1$ and $b_{i} \neq 1$, since those cases never occur in our construction of minimal Seifert surfaces for knots of even type. We remark that the assumption $a_{i} \neq-1, b_{i} \neq 1$ is essential in our proof of Theorem 6.2. At the end of this section, we briefly deal with this excluded case (see Theorem 6.5).

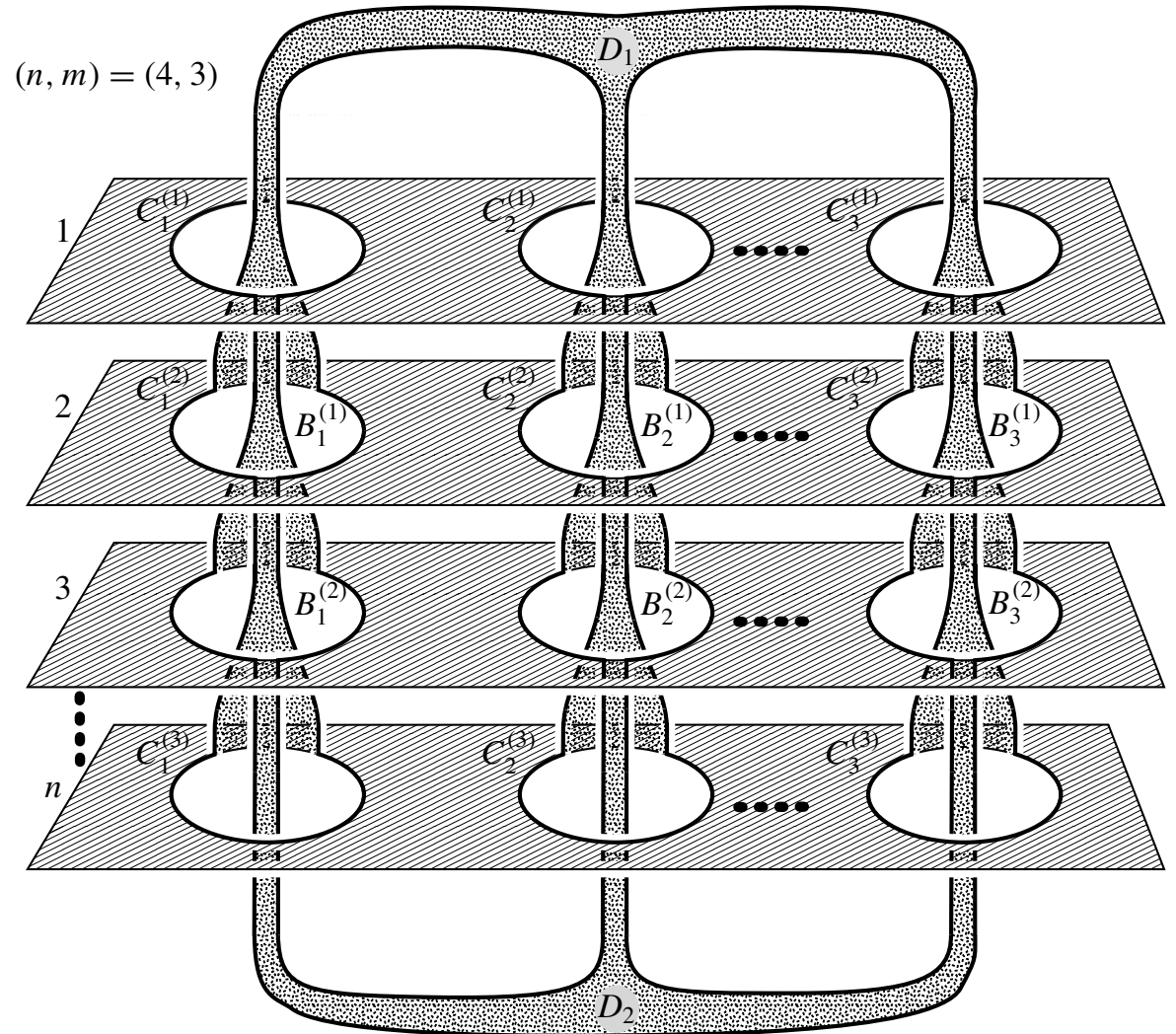

Figure 14. A minimal Seifert surface for $P(-(n+1), n+1, \ldots,-(n+1), n+1)$. 


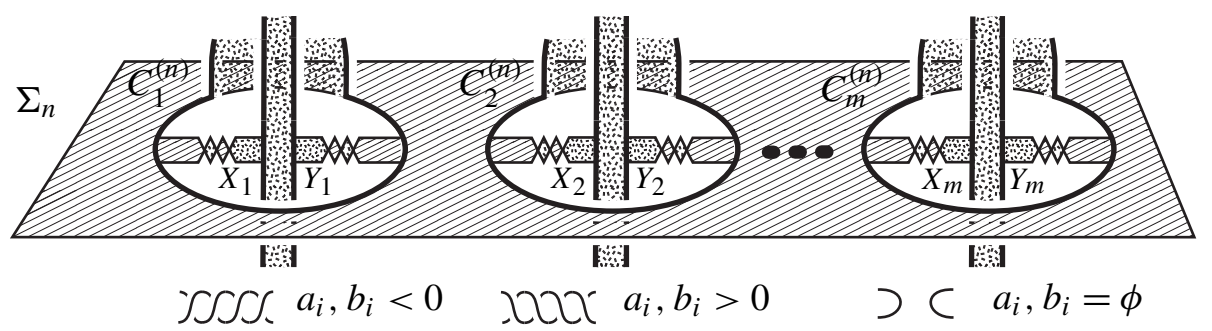

Figure 15. Additional bands.

Now the following proposition is straightforward.

Proposition 6.1. The Betti number of $F$ is given by

$$
\beta\left(F\left(n, m \mid\left(a_{1}, b_{1}\right),\left(a_{2}, b_{2}\right), \ldots,\left(a_{m}, b_{m}\right)\right)\right)=2(n+1)(m-1)-\lambda+1,
$$

where $\lambda$ is the number of $\phi$ 's in the sequence of pairs

$$
\left\{\left(a_{1}, b_{1}\right),\left(a_{2}, b_{2}\right), \ldots,\left(a_{m}, b_{m}\right)\right\} .
$$

Note. Compare $F(n, m)$ with the Seifert surface $H$ depicted in Figure 16 for the same oriented link $(n=4)$. Actually $F(n, m)$ is obtained from $H$ by compressing $n+1$ times. Two compressing disks for $H$ are easy to find, and a third appears after a compression.

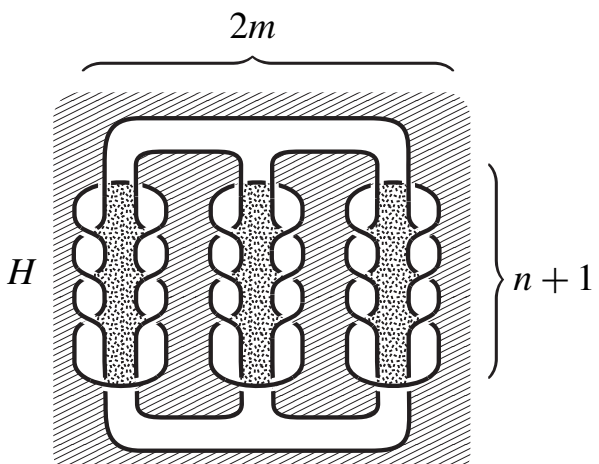

Figure 16. A compressible surface for $P(-(n+1), n+1, \ldots,-(n+1), n+1)$.

Here is the main theorem in this section:

Theorem 6.2. Let $F$ be the surface $F\left(n, m \mid\left(a_{1}, b_{1}\right),\left(a_{2}, b_{2}\right), \ldots,\left(a_{m}, b_{m}\right)\right)$ such that $a_{i} \neq-1$ and $b_{i} \neq 1$ for all $i$.

(I) $F$ is compressible if and only if all pairs $\left(a_{i}, b_{i}\right)$ are $(1,-1)$. Otherwise, $F$ is a minimal genus Seifert surface for $\partial F$.

(II) $F$ is a fibre surface if and only if all pairs are $(1,-1)$ except one pair which is one of four pairs: $(1, \phi),(1,-3),(\phi,-1)$ and $(3,-1)$. 
Under the additional condition that

$$
a_{i} \in\{1, \phi\} \quad \text { and } \quad b_{i} \in\{-1, \phi\} \quad \text { for all } i,
$$

Gabai proved, using sutured manifolds, that $F$ is of minimal genus if and only if not all pairs are $(1,-1)$, and that $F$ is fibered if and only if there is exactly one $a_{j}$ (or $b_{j}$ ) that is $\phi$. (See [Gabai 1986a, Theorem 6.7, Case 3] and its proof.) Gabai's surface looks a bit different, but after applying obvious deplumbing of Hopf bands in [Gabai 1986a, Figure 6.7(d-e)], his surface is isotopic to the mirror image of ours under condition (11).

For the fibredness of $F$, we give a simple proof by plumbing and deplumbing of Hopf bands.

Proof of the compressibility and fibredness in Theorem 6.2. Denote by $F\left(a_{1}\right)$ the special surface $F\left(n, m \mid\left(a_{1},-1\right),(1,-1), \ldots,(1,-1)\right)$.

First we prove $F(1)$ is compressible. The loop $\ell$ depicted in Figure 17 is trivial (i.e, bounds an embedded disk) in the complement of $F(1)$. On the other hand, we can push $\ell$ onto $F(1)$. Therefore, we can regard $\ell$ as (a slight push off of) the boundary of a compressing disk for $F(1)$.

To prove fibredness, it suffices to show that $F(\phi)$ and $F(3)$ are fibred (for the other two cases are similar). As we have just seen, $F(1)$ is compressible, where the loop $\ell$ runs once along the band $X_{1}$. Therefore, we see that $F(1)$ is obtained from $F(\phi)$ by plumbing a flat annulus $A(0)$, with $\ell$ regarded as the core loop (recall the deplumbing lemma). Replace the deplumbed flat annulus by a Hopf band $A(2)$ or $A(-2)$ and plumb it back to $F(\phi)$, to obtain $F(3)$ or $F(-1)$ respectively. By Theorem 2.9, all of $F(3), F(\phi), F(-1)$ are fibre surfaces (for different links) if and only if one of them is a fibre surface. We use $F(-1)$, though it is precluded in our theorem.

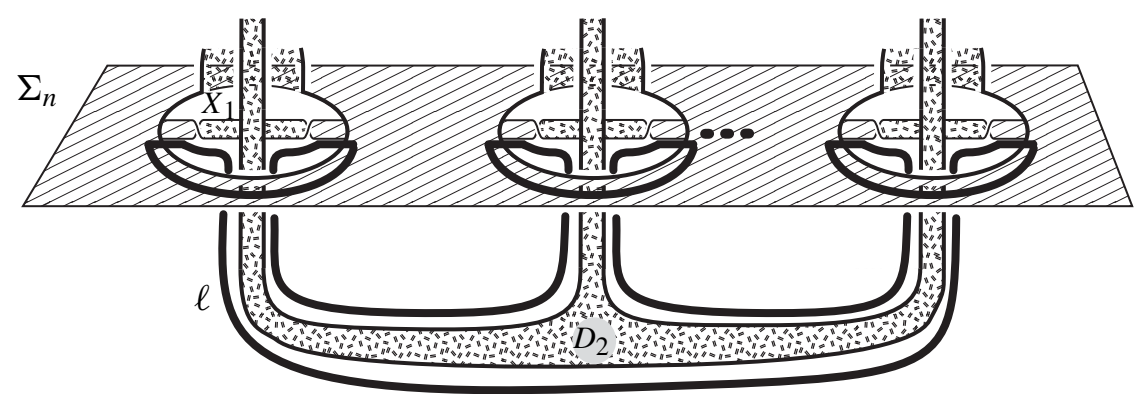

Figure 17. A push off of the boundary of a compressing disk. 
To $F(-1)$, we repeatedly plumb $n$ Hopf bands $A(-2)$ so that we multiply the -1 twisted band $X_{1} n+1$ times as in Figure 18, and denote the resulting surface by $F^{\prime}(-1)$.

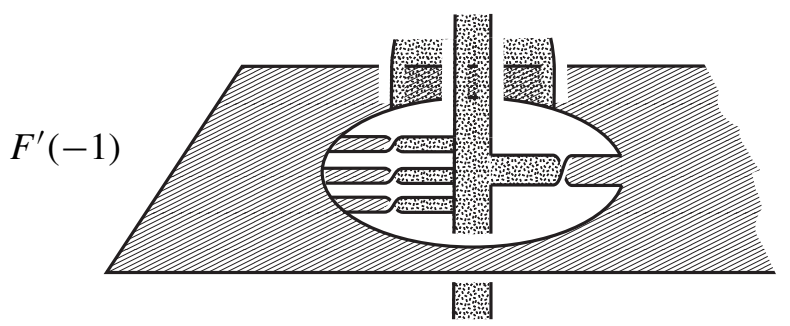

Figure 18. Multiplying the band $X_{1}$ by $n+1$.

Now we look at the diagram of the link itself. First, $\partial F(n, m \mid(\phi, \phi), \ldots,(\phi, \phi))$ in Figure 14 depicts $P(-(n+1), n+1, \ldots,-(n+1), n+1)$, and hence $\partial F(1)=$ $P(-(n+2), n+2, \ldots,-(n+2), n+2)$. Also, we can directly see that $\partial F(-1)=$ $P(-(n+1)+1, n+2, \ldots,-(n+2), n+2)$ and hence $\partial F^{\prime}(-1)=P(-(n+1)+$ $1+n, n+2, \ldots,-(n+2), n+2)=P(0, n+2, \ldots,-(n+2), n+2)$.

On the other hand, the natural pretzel link $P(0, n+2, \ldots,-(n+2), n+2)$ spans the Seifert surface $G$ depicted in Figure 19. Since $G$ is a plumbing of Hopf bands, $G$ is a fibre surface.

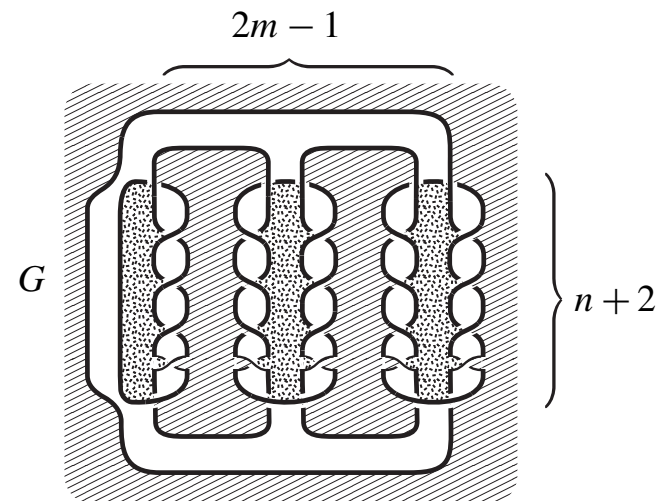

Figure 19. A fibre surface that is a plumbing of Hopf bands.

Furthermore, by the following calculation, we see that $G$ and $F^{\prime}(-1)$ have the same Betti number. $\beta\left(F^{\prime}(-1)\right)=\beta(F(-1))+n=2(n+1)(m-1)+1+n=$ $(2 m-1)(n+1)=\beta(G)$. (The second equality is obtained by Proposition 6.1 with $\lambda=0$.)

Since $\partial G=\partial F^{\prime}(-1)$ as an oriented link, we see, by the uniqueness of fibre surfaces for a fibred link, $G$ is isotopic to $F^{\prime}(-1)$.

Therefore, $F^{\prime}(-1)$ is a fibre surface, and hence by Theorem 2.9, $F(-1), F(\phi)$, $F(3)$ are all fibre surfaces. 


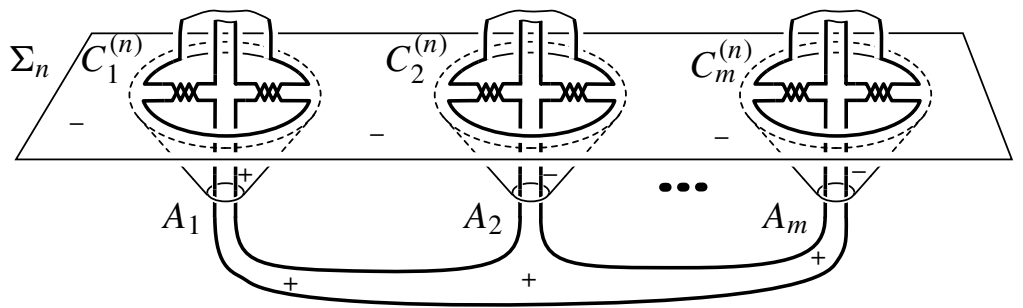

Figure 20. Sutured manifold decompositions using annuli.

Note. It is possible to obtain an inductive picture of the isotopy between $G$ and $F^{\prime}(-1)$.

Proof of minimality of the genus in Theorem 6.2. In the remainder of this section, we use sutured manifolds and sutured manifold decompositions. For basic definitions and facts, see [Gabai 1986b, pp. 8-10 and Appendix A] and [Gabai 1986a, Section 1].

Let $F$ be the surface $F\left(n, m \mid\left(a_{1}, b_{1}\right),\left(a_{2}, b_{2}\right), \ldots,\left(a_{m}, b_{m}\right)\right)$ such that $a_{i} \neq-1$ and $b_{i} \neq 1$ for each $i$. Suppose that not all pairs of $\left(a_{1}, b_{1}\right),\left(a_{2}, b_{2}\right), \ldots,\left(a_{m}, b_{m}\right)$ are $(1,-1)$. In particular, we assume that at least $\left(a_{1}, b_{1}\right)$, as an exceptional pair, is not $(1,-1)$.

Our strategy, like Gabai's [1986a, Theorem 6.7, Case 3], is to find a sutured manifold hierarchy (i.e., a sequence of sutured manifold decompositions reducing the complementary sutured manifold of a surface to a union of 3-balls each of which has a single suture).

Let $A_{1}, \ldots, A_{m}$ be annuli in Figure 20 with specific orientations as depicted, where $A_{1}$ shows the opposite side than $A_{2}, \ldots A_{m}$. The first step of our decomposition uses these annuli.

The complementary sutured manifold of $F$ splits into two components, say $(M, \gamma)$ lying above $\Sigma_{n}$ and $(N, \delta)$ lying below $\Sigma_{n}$. Figure 21 depicts $(N, \delta)$ viewed from below (hence $\Sigma_{n}$ shows a different side than in Figure 20). It is easy to see that $(N, \delta)$ is a product (and hence decomposable) sutured manifold. Therefore, it suffices to show that $(M, \gamma)$ is decomposable.

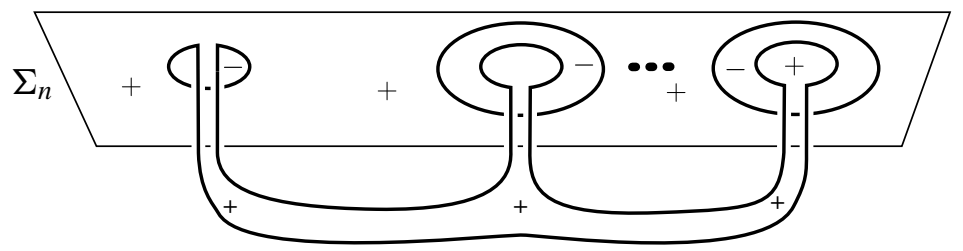

Figure 21. Product sutured manifold below $\Sigma_{n}$. 
Near the holes by $C_{i}^{(n)},(M, \gamma)$ locally looks as shown in the two parts of Figure 22. The horizontal band may be missing if its corresponding entry $a_{i}$ or $b_{i}$ is $\phi$.

First, we consider pairs $\left(a_{i}, b_{i}\right)$ 's with $i \geq 2$. In Figure 22, top, apply an annulus decomposition and then at most two disc decompositions as depicted. Denote the result by $\left(M^{\prime}, \gamma^{\prime}\right)$. (The assumptions $a_{i} \neq-1, b_{i} \neq 1$ are essential here.)

Next, consider the exceptional pair $\left(a_{1}, b_{1}\right)$ in Figure 22, bottom. If $a_{1}=1$, we can apply a product decomposition to $\left(M^{\prime}, \gamma^{\prime}\right)$ as in Figure 23(a1). Likewise, if $b_{1}=-1$, see Figure 23(b1). In the case $\left|a_{1}\right|,\left|b_{1}\right|>1$, we have two paths for the disk decompositions, as in the rest of Figure 23.

Now, by at most two decompositions, $\left(M^{\prime}, \gamma^{\prime}\right)$ is locally modified as in one of the three cases of Figure 24, which we denote respectively by $\left(M_{1}, \gamma_{1}\right),\left(M_{\phi}, \gamma_{\phi}\right)$ and $\left(M_{-1}, \gamma_{-1}\right)$.

Now we show that $\left(M_{1}, \gamma_{1}\right),\left(M_{\phi}, \gamma_{\phi}\right)$ and $\left(M_{-1}, \gamma_{-1}\right)$ are obtained from certain decomposable sutured manifolds by product decompositions. Then by [Gabai 1986a, Proposition A.5], they are decomposable and hence our $F$ is of minimal genus.

Namely, we show:

Lemma 6.3. Denote by $R\left(a_{1}, b_{1}\right)$ the surface $F\left(n, m \mid\left(a_{1}, b_{1}\right),(1,-1),(1,-1)\right.$, $\ldots,(1,-1))$. Then $\left(M_{1}, \gamma_{1}\right),\left(M_{\phi}, \gamma_{\phi}\right)$ and $\left(M_{-1}, \gamma_{-1}\right)$ are respectively obtained by applying product decompositions to the complementary sutured manifolds of $R(1, \phi), R(\phi, \phi)$ and $R(\phi,-1)$.

Note that $R(1, \phi), R(\phi, \phi)$ and $R(\phi,-1)$ have been shown to be of minimal genus [Gabai 1986a, Theorem 6.7, Case 3]. (We can also see directly that $R(1, \phi)$
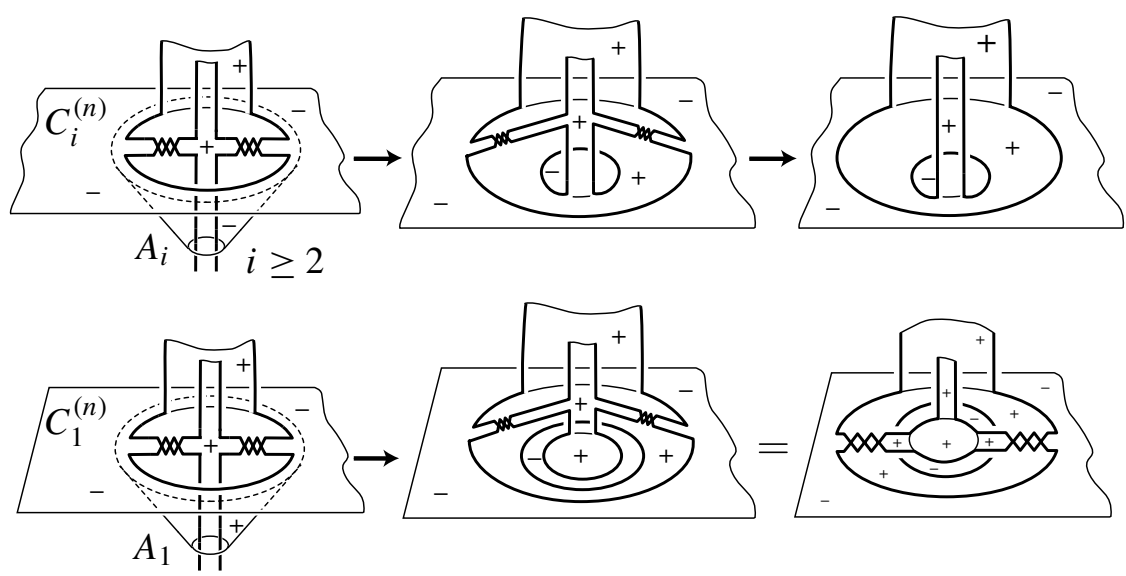

Figure 22. Sutured manifold $(M, \gamma)$. Top: $i \geq 2$; bottom: $i=1$. 

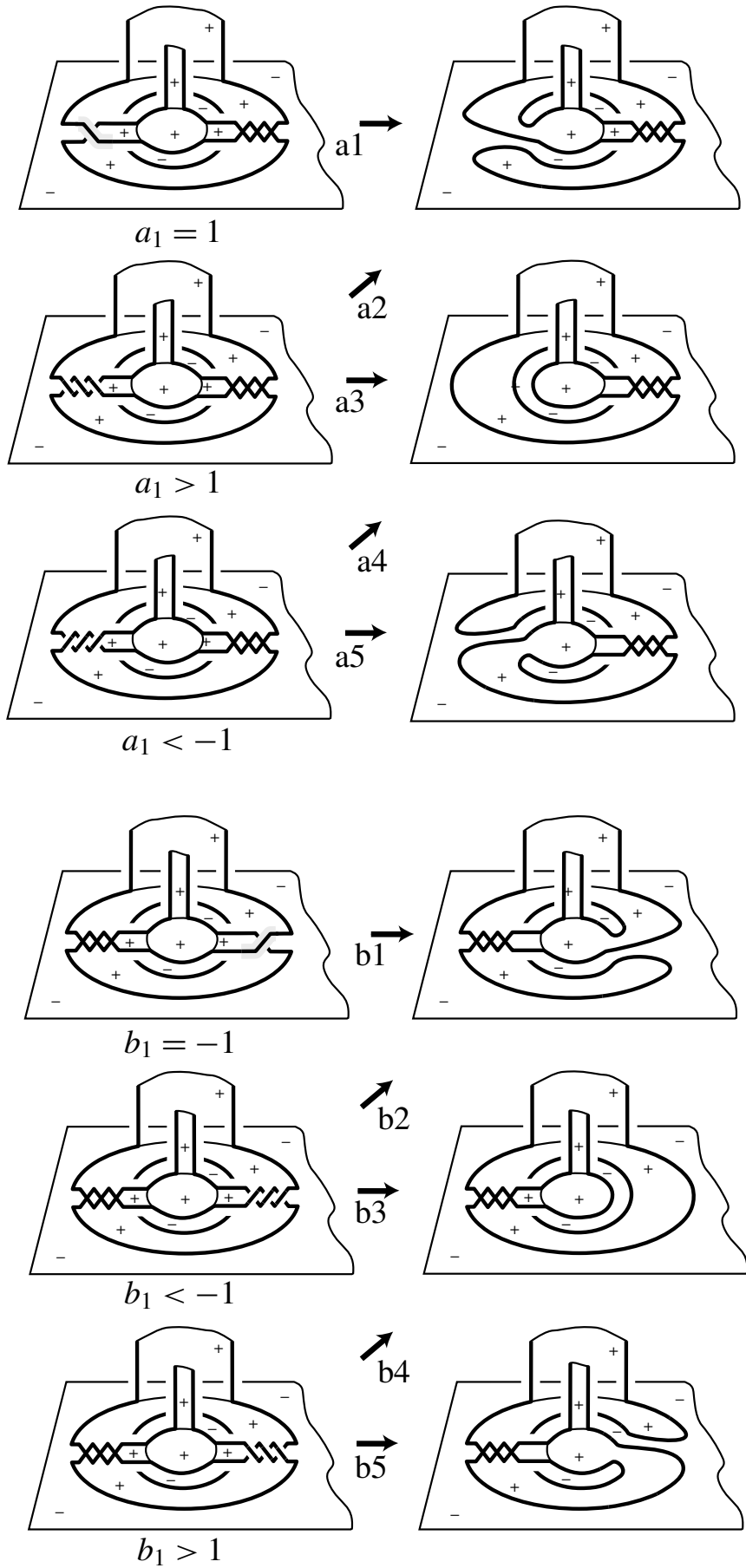

Figure 23. The sutured manifold decomposition near $C_{1}^{(n)}$. 


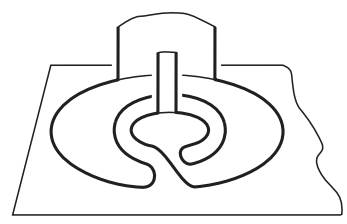

$\left(M_{-1}, \gamma_{-1}\right)$

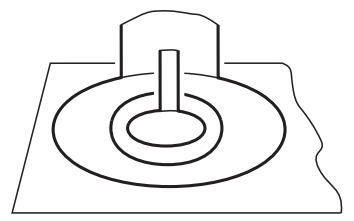

$\left(M_{\phi}, \gamma_{\phi}\right)$

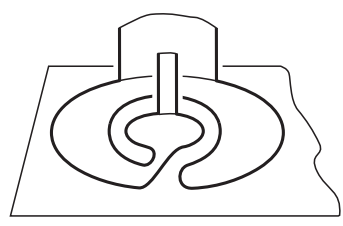

$\left(M_{1}, \gamma_{1}\right)$

Figure 24. $\left(M^{\prime}, \gamma^{\prime}\right)$ is decomposed into $\left(M_{1}, \gamma_{1}\right),\left(M_{\phi}, \gamma_{\phi}\right)$ or $\left(M_{-1}, \gamma_{-1}\right)$.

and $R(\phi,-1)$ are so, since they are fibre surfaces.) Therefore, complementary sutured manifolds of these surfaces are decomposable [Gabai 1986a, Remark 1.31]. Proof of Lemma 6.3. Since other cases are similar, we only consider the case of $R(1, \phi)$. Take the complementary sutured manifold for $R(1, \phi)$ and apply two product decompositions at each hole by $C_{i}^{(n)}, i \geq 2$ as in Figure 25, where the loop indicates the first decomposition.

Then we amalgamate the handles below (an image of) $\Sigma_{n}$ by product decompositions. Finally, by a product decomposition as in Figure 23(a1), we obtain $\left(M_{1}, \gamma_{1}\right)$.

This concludes the proof that the genus is minimal.

Proof of the nonfibredness in Theorem 6.2. First suppose that there is another exceptional pair, say $\left(a_{j}, b_{j}\right)$. That is, $\left(a_{1}, b_{1}\right) \neq(1,-1)$ and $\left(a_{j}, b_{j}\right) \neq(1,-1)$. Then, as in [Gabai 1986a, p. 546], we see that $F$ is decomposable "in two ways", and hence $F$ is not a fibre surface [Gabai 1986a, Corollary 2.7]. To be more precise, the complementary sutured manifold of $F$ has two sutured manifold hierarchies; one of them starts with the decomposition using the surface $S=A_{1} \cup A_{j}$ with the orientation specified in Figure 20, and the other starts with the decomposition using $S$ with the opposite orientation (and afterwards proceeds as before).

Finally, suppose that there is only one exceptional pair, that is, assume that $\left(a_{1}, b_{1}\right) \neq(1,-1)$ and $\left(a_{i}, b_{i}\right)=(1,-1)$ for all $i \geq 2$.

Case 1: $a_{1} \neq 1$ and $b_{1} \neq-1$. If $\left(a_{1}, b_{1}\right)=(\phi, \phi)$, then $F$ is not a fiber surface by [Gabai 1986a, Theorem 6.7, Case 3].

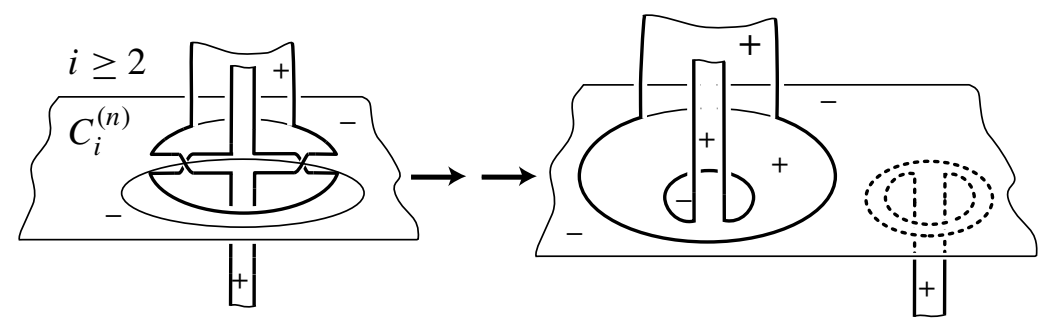

Figure 25. Two product decompositions at each $i$-th hole $(i \geq 2)$. 

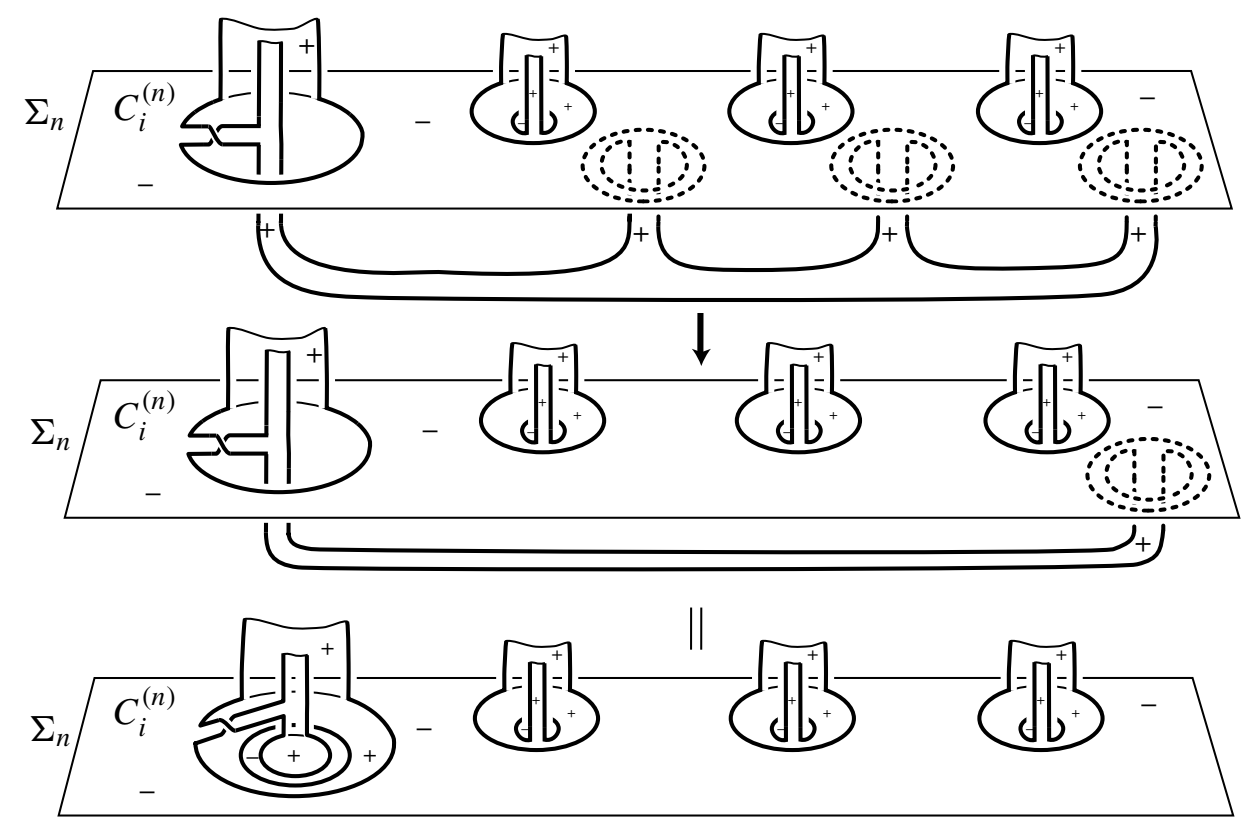

Figure 26. Amalgamation of handles by product decompositions.

So, suppose $\left(a_{1}, b_{1}\right) \neq(\phi, \phi)$. We have seen that sutured manifolds in Figure 24 are decomposable. Therefore, if we can decompose the part in Figure 22, bottom, as in Figure 24, we can complete the sutured manifold hierarchy. Then we see, as below by using Figure 23, that we have two paths for hierarchies (and hence that $F$ is not a fibre surface): namely, If $a_{1}=\phi$, pretend that $b_{1}=\operatorname{sign}\left(b_{1}\right)$ or $b_{1}=\phi$. If $b_{1}=\phi$, pretend that $a_{1}=\operatorname{sign}\left(a_{1}\right)$ or $a_{1}=\phi$. If neither of $a_{1}, b_{1}$ is $\phi$, pretend that $\left(a_{1}, b_{1}\right)=\left(\operatorname{sign}\left(a_{1}\right), \phi\right)$ or $\left(\phi, \operatorname{sign}\left(b_{i}\right)\right)$. In each case, we have one of the possibilities of Figure 24.

Case 2: Either $a_{1}=1$ or $b_{1}=-1$. We only deal with the case $b_{1}=-1$; the other case is analogous. As before, set $F\left(a_{1}\right):=F\left(n, m \mid\left(a_{1},-1\right),(1,-1), \ldots,(1,-1)\right)$. We already know that $F\left(a_{1}\right)$ is of minimal genus, since $a_{1} \neq 1$. Deplumb the annulus $A\left(a_{1}-1\right)$ from $F\left(a_{1}\right)$ as before. Since $A\left(a_{1}-1\right)$ is a fibre surface if and only if $a_{1}-1= \pm 2$, we see by Theorem 2.9 that $F\left(a_{1}\right)$ is not a fibre if $a_{1} \neq-1$ or $\neq 3$.

Remark 6.4. In Theorem 6.2, we assumed $a_{i} \neq-1$ and $b_{i} \neq 1$ for all $i$. We call the band a forbidden band if the corresponding entry is $a_{i}=-1$ or $b_{i}=1$. Suppose that $X_{1}$ is a forbidden band. By finding a compressing disk and plumbing and deplumbing Hopf bands, we can prove: 
Theorem 6.5. Let $F:=F\left(n, m \mid\left(a_{1}, b_{1}\right),\left(a_{2}, b_{2}\right), \ldots,\left(a_{m}, b_{m}\right)\right)$ be such that $X_{1}$ is a forbidden band, i.e., $a_{1}=-1$.

(I) $F$ is compressible if and only if there is another forbidden band. Otherwise, $F$ is a minimal genus Seifert surface for $\partial F$.

(II) $F$ is a fibre surface if and only if all the other $a_{i}$ 's $(i \geq 2)$ equal $1, \phi$ or -3 , and all the $b_{i}$ 's $(i \geq 1)$ equal $-1, \phi$ or 3 .

\section{Proof of Theorem 3.2(II)}

We now consider the exceptional case that was not dealt with in Section 5.

Let $S_{i}=\left[2 c_{1}{ }^{(i)}, 2 c_{2}{ }^{(i)}, 2 c_{3}{ }^{(i)}, \ldots, 2 c_{m_{i}}{ }^{(i)}\right]$ be an even continued fraction of $\frac{\beta_{i}}{\alpha_{i}}$, for $1 \leq i \leq r$.

It suffices to consider the case

$$
\left(c_{1}^{(1)}, c_{1}{ }^{(2)}, \ldots, c_{1}{ }^{(r)}\right)=(1,-1, \ldots, 1,-1) \quad \text { and } e=0 .
$$

Define $p_{i}$ as the number of leading 2's in $S_{i}$, if $i$ is odd, or the number of leading -2 's in $S_{i}$, if $i$ is even. Set $p=\min \left\{p_{1}, p_{2}, \ldots, p_{r}\right\}$. Then $p \geq 1$, by assumption (12).

Now using (7) or (8), we can write, for odd $i, S_{i}=1+\left[-(p+1), 2 c_{p+1}{ }^{(i)}-1\right.$, $\left.2 c_{p+2}{ }^{(i)}, \ldots, 2 c_{m_{i}}{ }^{(i)}\right]$, and for even $i, S_{i}=-1+\left[p+1,2 c_{p+1}{ }^{(i)}+1,2 c_{p+2}{ }^{(i)}, \ldots\right.$, $2 c_{m_{i}}{ }^{(i)}$ ]. Recalling Remark 2.2, we merge the 1's and -1 's arisen in the rewriting, and obtain a new diagram $D$ as in Figure 27.

Apply the Seifert algorithm on $D$ to obtain a Seifert surface $F$. However, $F$ is compressible. In order to obtain a Seifert surface of minimal genus, we cut $F$ into two parts $F_{1}$ and $F_{2}$ along a horizontal broken line in Figure 27. In fact $F_{1}$ in Figure 28 is a compressible surface, and therefore, we replace $F_{1}$ by the surface $\widetilde{F}_{1}=F\left(p, \frac{r}{2}\right)$ introduced in Section 6 . Join $F_{2}$ to $\widetilde{F}_{1}$ (at the original places)to obtain a new Seifert surface $\widetilde{F}=\widetilde{F}_{1} \cup F_{2}$ for the original knot $K$.

To show that $\widetilde{F}$ is of minimal genus, first we deplumb $\left(m_{i}-(p+1)\right)$ annuli $A\left(2 c_{j}{ }^{(i)}\right), p+2 \leq j \leq m_{i}$, from each tangle $T\left(\frac{\beta_{i}}{\alpha_{i}}\right)$, and, in total, we deplumb $\sum_{i=1}^{r}\left(m_{i}-(p+1)\right)$ annuli $A\left(2 c_{j}{ }^{(i)}\right)$, with $1 \leq i \leq r$ and $p+2 \leq j \leq m_{i}$. What is left is exactly the surface

$$
\begin{array}{r}
F^{*}=F\left(p, \frac{r}{2} \mid\left(2 c_{p+1}{ }^{(1)}-1,2 c_{p+1}{ }^{(2)}+1\right),\left(2 c_{p+1}{ }^{(3)}-1,2 c_{p+1}^{(4)}+1\right), \ldots,\right. \\
\left.\left(2 c_{p+1}{ }^{(r-1)}-1,2 c_{p+1}{ }^{(r)}+1\right)\right) .
\end{array}
$$

We recall that some pair may contain $\phi$.

Now by construction, it is impossible that every pair is $(1,-1)$. Moreover, since $2 c_{p+1}^{(i)} \neq 0$, we have $2 c_{p+1}^{(i)} \pm 1 \neq \pm 1$. Therefore, by Theorem $6.2, F^{*}$ is of minimal genus, and $\beta\left(F^{*}\right)=2(p+1)\left(\frac{r}{2}-1\right)-\lambda+1+\sum_{i=1}^{r}\left(m_{i}-(p+1)\right)+\lambda=$ 

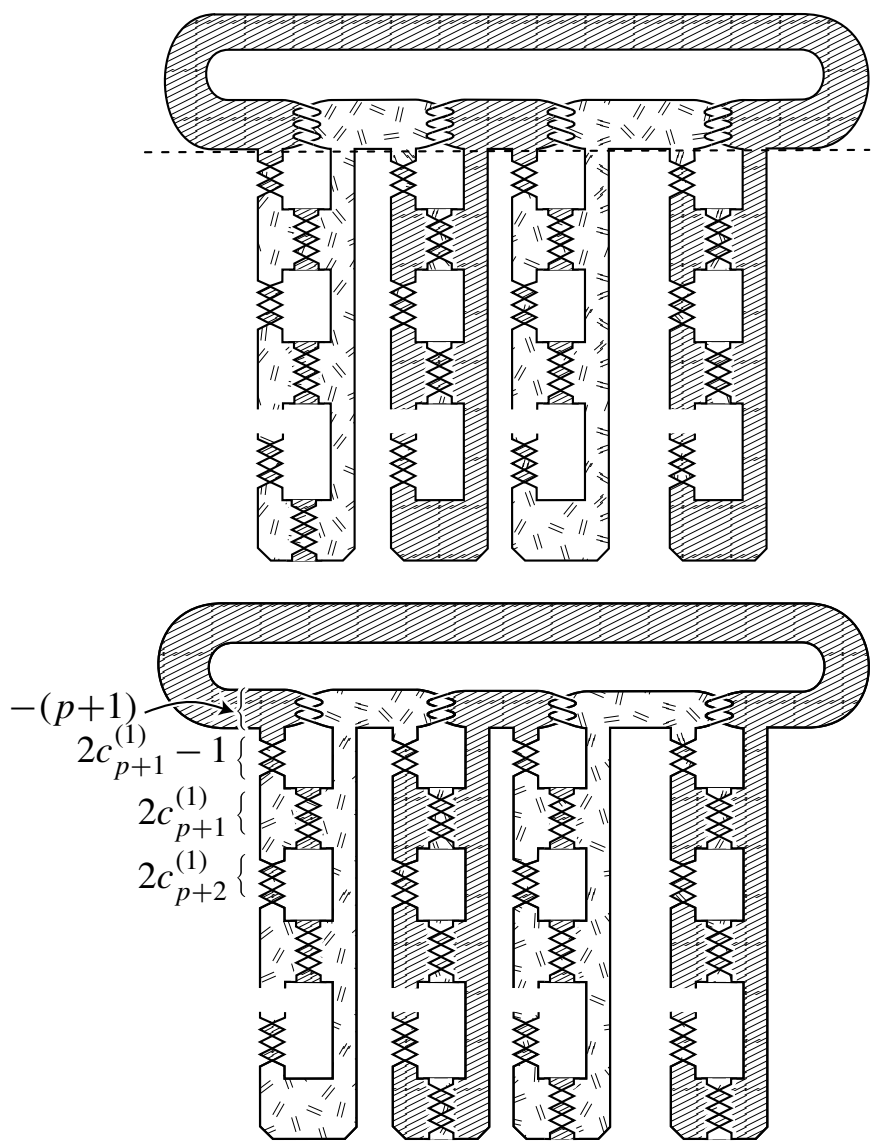

Figure 27. Compressible Seifert surfaces, when $p$ is odd (top) or even (bottom).

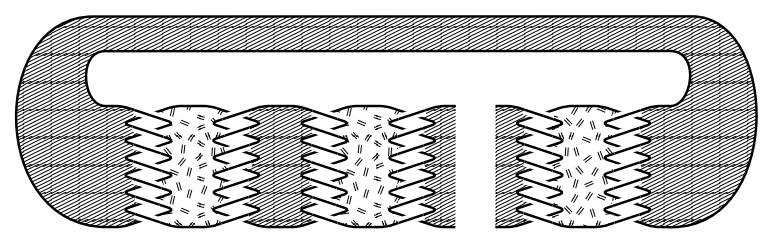

Figure 28. A compressible Seifert surface for $P(-(p+1), p+1$, $\ldots,-(p+1), p+1)$.

$\sum_{i=1}^{r} m_{i}-2(p+1)+1$; hence the genus of $K$ is

$$
g(K)=\frac{1}{2}\left(\sum_{i=1}^{r} m_{i}+1\right)-(p+1) .
$$

This proves (III-1). 
The fibredness conditions for $F$, and in particular those for $F^{*}$ given in Theorem 6.2 are equivalent to (III-ii). The proof of Theorem 3.2 is now complete.

Example 7.1. (1) Consider $K=M\left(\frac{3}{4},-\frac{8}{11}, \frac{2}{3},-\frac{8}{11} \mid 0\right)$. Since $3 / 4=[2,2,2]$, $-8 / 11=[-2,-2,-2,2]$, and $2 / 3=[2,2]$, we see that

$$
\left(c_{1}^{(1)}, c_{1}^{(2)}, c_{1}^{(3)}, c_{1}^{(4)}\right)=(1,-1,1,-1),
$$

and hence apply Theorem 3.2(III). First, since $p_{1}=p_{2}=p_{4}=3$ and $p_{3}=2$, we have $p=2$, and then $g(K)=4$. Further, $\left\{\left(2 c_{3}^{(1)}-1,2 c_{3}^{(2)}+1\right),\left(2 c_{3}^{(3)}-1,2 c_{3}^{(4)}+1\right)\right\}=$ $\{(1,-1),(\phi,-1)\}$, and thus $K$ is a fibred knot.

(2) Consider $K=M\left(\frac{7}{10},-\frac{8}{11}, \frac{8}{11},-\frac{8}{11} \mid 0\right)$. Since $\frac{7}{10}=[2,2,4]$, we see that $p=2$ and $g(K)=5$ and $\left\{\left(2 c_{3}^{(1)}-1,2 c_{3}^{(2)}+1\right),\left(2 c_{3}^{(3)}-1,2 c_{4}^{(4)}+1\right)\right\}=\{(3,-1),(1,-1)\}$. Therefore, $K$ is a fibred knot.

Remark 7.2. For Montesinos knots of even type, it suffices to assume that $e$ is even. However, if we assume $e$ is odd, we can also depict a minimal genus Seifert surface obtained by the Seifert algorithm in most cases.

For $\beta_{1} / \alpha_{1}$ find a continued fraction $\left[a_{1}, b_{1}, \ldots, b_{m-1}, a_{m}\right]$ such that the $b_{i}$ 's are even and $b_{i} a_{i+1}<0$ if $b_{i}= \pm 2$. Such a continued fraction always exists. For $\beta_{j} / \alpha_{j}$, find an even continued fraction. Then using these continued fractions, we find a Seifert surface, as depicted in Figure 29.

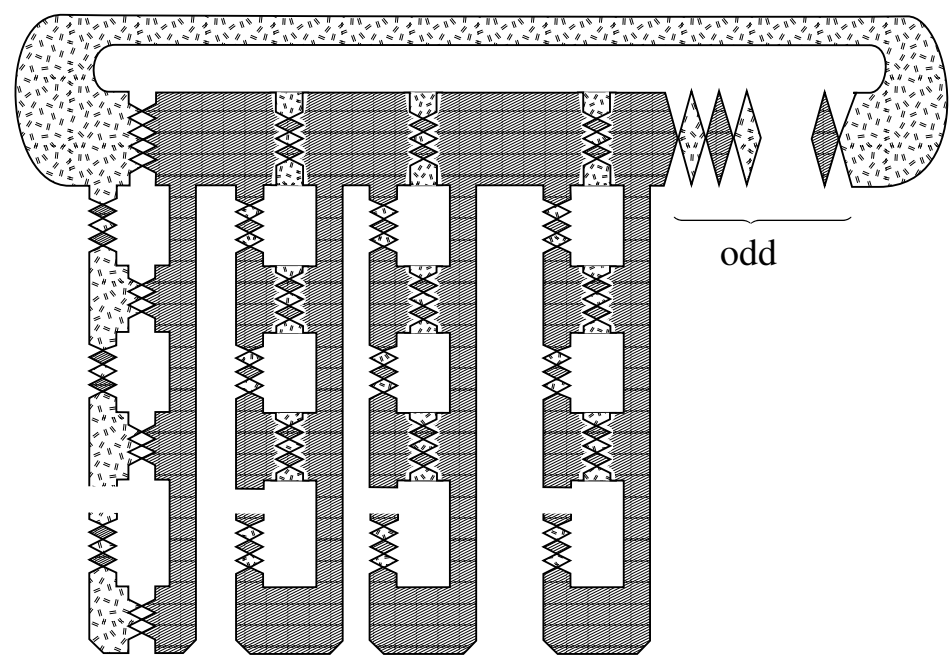

Figure 29. Another form of a minimal surface for knots of even type ( $e$ is odd and $\left.e \cdot \varepsilon\left(a_{1}\right)>0\right)$.

If $e+\varepsilon\left(a_{1}\right) \neq 0$, the Seifert surface in Figure 29 is of minimal genus. If $e+\varepsilon\left(a_{1}\right)=0$, assume $e=-1$. We obtain a new diagram and a surface as in Figure 30 , which is of a minimal Seifert surface at least if $\left(c_{1}^{(1)}, c_{1}^{(2)}, \ldots, c_{1}^{(r)}\right) \neq$ 


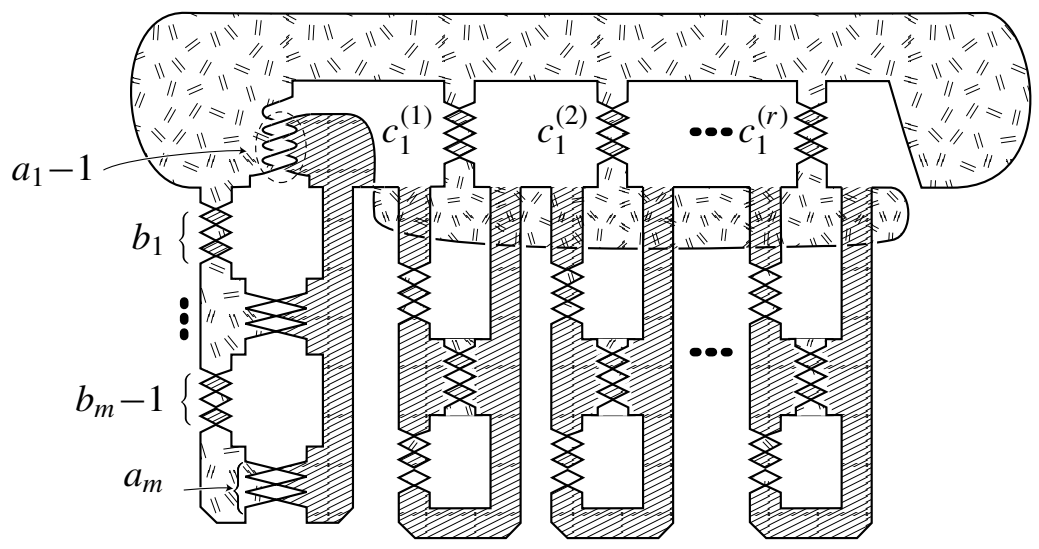

Figure 30. Another form of a minimal surface for knots of even type $\left(e+\varepsilon\left(\left(a_{1}\right)\right)=0\right.$ and $\left.e=-1\right)$.

$\pm(1,-1,1,-1, \ldots, 1)$. We omit the other cases, which are not needed in this paper.

\section{Tunnel number one Montesinos knots}

Montesinos knots of tunnel number one have been classified:

Theorem 8.1 [Morimoto et al. 1996; Klimenko and Sakuma 1998]. The Montesinos knot $K=M\left(\frac{\beta_{1}}{\alpha_{1}}, \frac{\beta_{2}}{\alpha_{2}}, \ldots, \frac{\beta_{r}}{\alpha_{r}} \mid e\right)$ has tunnel number one if and only if one of the following conditions is satisfied.

(1) $r=2$.

(2) $r=3, \beta_{2} / \alpha_{2} \equiv \beta_{3} / \alpha_{3} \equiv \pm \frac{1}{3}$ in $\mathbb{Q} / \mathbb{Z}$, and $e+\sum_{i=1}^{3} \beta_{i} / \alpha_{i}= \pm 1 /\left(3 \alpha_{1}\right)$.

(3) $r=3, \alpha_{2}$ and $\alpha_{3}$ are odd, and $\alpha_{1}=2$.

(Theorem 8.1 differs from [Klimenko and Sakuma 1998, Corollary C] in the sign conventions for $e$.)

To determine fibred Montesinos knots of tunnel number one in terms of their Alexander polynomials, we consider a slightly wider class of knots. (However, we exclude the case $r=2$, since in that case, $K$ is a 2-bridge knot.) Our class consists of the following three subclasses.

Class (1): $\alpha_{1}$ is odd, $\beta_{2} / \alpha_{2}=\beta_{3} / \alpha_{3}= \pm \frac{1}{3}$, and $e$ is arbitrary.

Class (2): $\alpha_{1}$ is even, $\alpha_{1} \geq 4, \beta_{2} / \alpha_{2}=\beta_{3} / \alpha_{3}= \pm \frac{1}{3}$, and $e$ is even.

Class (3): $\beta_{1} / \alpha_{1}=\frac{1}{2}$, both $\alpha_{2}$ and $\alpha_{3}$ are odd ( $\left.\geq 1\right)$, and $e$ is even.

By Proposition 2.3, it is evident that our class contains all tunnel number one Montesinos knots (but 2-bridge knots).

A knot in class (1) is of odd type, while a knot in class (2) or (3) is of even type. 
Proposition 8.2. Let $F$ be a minimal genus Seifert surface for a knot $K$, and let $F_{0}$ be a surface obtained from $F$ by deplumbing an annulus $A$. Suppose that the Alexander polynomial $\Delta_{K}(t)$ of $K$ is monic, i.e. $\left|\Delta_{K}(0)\right|=1$. Further, we assume that

$$
\text { the degree of } \Delta_{K}(t) \text { equals } 2 g(K) \text {. }
$$

Then $A$ is a Hopf band, and $\Delta_{K_{0}}(t)$ is monic, where $K_{0}=\partial F_{0}$.

Proof. Let $M$ be the Seifert matrix for $K$ obtained from $F$. By assumption (13), $M$ is nonsingular and $|\operatorname{det} M|=\left|\Delta_{K}(0)\right|$. This proves that $A$ is a Hopf band, since otherwise we would have $\operatorname{det} M \neq \pm 1$.

Now let $M_{0}$ be a Seifert matrix for $K_{0}$ obtained from $F_{0}$. Again by (13), $|\operatorname{det} M|=\left|\operatorname{det} M_{0}\right|=1$. Since $\left|\operatorname{det} M_{0}\right|=\left|\Delta_{K_{0}}(0)\right|$, the rest of the proposition follows.

Let $V_{1}$ and $V_{2}$, respectively, be Seifert matrices for the natural pretzel links $P\left(2 p_{1}+1,2 p_{2}+1, \ldots, 2 p_{n}+1\right)$ and $P\left(2 p_{1}, 2 p_{2}, \ldots, 2 p_{n}\right)$. It is known (see [Kanenobu 1979, pp. 368, 372], for example) that

and

$$
\operatorname{det} V_{1}=\prod_{i=1}^{n}\left(p_{i}+1\right)-\prod_{i=1}^{n} p_{i}
$$

$$
\operatorname{det} V_{2}=p_{1} p_{2} \cdots p_{n} \sum_{i=1}^{n} \frac{1}{p_{i}} \text {. }
$$

Using these formulae, we can determine the fibred Montesinos knots of tunnel number one in terms of their Alexander polynomials.

Theorem 8.3. Let $K$ be a Montesinos knot in class (1) or class (2). If the Alexander polynomial of $K$ is monic, then $K$ is fibred.

Proof. (i) Let $K$ be a Montesinos knot in class (1). We may assume, by taking the mirror image if necessary, that $K=M\left(\frac{\beta_{1}}{\alpha_{1}}, \frac{1}{3}, \frac{1}{3} \mid e\right)$, where $\alpha_{1}$ is odd $(>1)$ and $0<2\left|\beta_{1}\right|<\alpha_{1}$.

Let $S_{1}=\left[2 a_{1}, b_{1}, 2 a_{2}, b_{2}, \ldots, 2 a_{q}, b_{q}\right]$, and $S_{2}=S_{3}=[2,-1]$ be strict continued fractions of $\frac{\beta_{1}}{\alpha_{1}}$, and $\frac{1}{3}$, respectively. We apply the proof of Theorem 3.1 to our knot. Then $\partial F_{0}$ is the natural pretzel link $K_{0}=P\left(2 a_{1}-\varepsilon\left(b_{1}\right), 3,3, \varepsilon(e), \ldots, \varepsilon(e)\right)$. Note that $\left|2 a_{1}-\varepsilon\left(b_{1}\right)\right| \geq 3$. Then using (14), we see easily that the Seifert matrix $M_{0}$ of $K_{0}$ is not singular and that (13) is satisfied. Now $\Delta_{K_{0}}(t)$ is monic if and only if $2 a_{1}-\varepsilon\left(b_{1}\right)=3$ and $e<0$. Therefore, by Theorem 3.1, $F_{0}$ is a fibre surface if $\Delta_{K_{0}}(t)$ is monic, and hence $K$ is a fibred knot. 
(ii) Consider a knot in class (2). We may assume that $K$ is of the form

$$
M\left(\frac{\beta_{1}}{\alpha_{1}},-\frac{2}{3},-\frac{2}{3} \mid e\right),
$$

where $\alpha_{1}$ is even, $\alpha_{1} \geq 4$ and $e$ is even.

Let $S_{1}=\left[2 c_{1}, 2 c_{2}, \ldots, 2 c_{m}\right]$ and $S_{2}=S_{3}=[-2,-2]$ be the even continued fractions of $\frac{\beta_{1}}{\alpha_{1}}$ and $-\frac{2}{3}$, respectively. Suppose that $\Delta_{K}(t)$ is monic.

(I) Suppose $e \neq 0$.

We apply the proof of Theorem 3.2 in Section 5 on $K$. Then $F_{0}$ is an annulus $A(e)$. Thus condition (13) is satisfied and, since $\Delta_{K}(t)$ is monic, it follows that $\left|c_{1}\right|=\left|c_{2}\right|=\cdots=\left|c_{m}\right|=1$ by Proposition 8.2, and further, $|e|=2$. Therefore, $K$ is fibred.

(II) Suppose $e=0$. Then $\partial F_{0}=K_{0}$ is the pretzel link $P\left(2 c_{1},-2,-2\right)$. Let $M_{0}$ be a Seifert matrix of $K_{0}$. Then $\operatorname{det} M_{0}=-2 c_{1}+1 \neq 0$ and hence, (13) is satisfied. Since $\Delta_{K}(t)$ is monic, we have $\left|c_{2}\right|=\left|c_{3}\right|=\cdots=\left|c_{m}\right|=1$. Further, $\left|\operatorname{det} M_{0}\right|=1$ if and only if $c_{1}=1$. Therefore, if $\Delta_{K}(t)$ is monic, then $K_{0}$ is fibred and hence $K$ is fibred.

Now we turn to class (3). This case is exceptional, in that there exist nonfibred knots with monic Alexander polynomials.

Let $K=M\left(\frac{1}{2}, \frac{\beta_{2}}{\alpha_{2}}, \frac{\beta_{3}}{\alpha_{3}} \mid e\right)$ be a knot in class (3). Consider the even continued fractions $\frac{1}{2}=[2], \beta_{2} / \alpha_{2}=\left[2 x_{1}, 2 x_{2}, \ldots, 2 x_{p}\right]$ and $\beta_{3} / \alpha_{3}=\left[2 y_{1}, 2 y_{2}, \ldots, 2 y_{q}\right]$. Assume that $\Delta_{K}(t)$ is monic.

(I) Suppose $e \neq 0$. Since $\Delta_{K}(t)$ is monic, we have $\left|x_{i}\right|=\left|y_{j}\right|=1$ for $1 \leq i \leq p$ and $1 \leq j \leq q$. Further, we showed in the proof of Theorem 8.3 that $F_{0}$ is an annulus $A(e)$, and hence if $\Delta_{K}(t)$ is monic, then $K$ is fibred.

(II) Suppose $e=0$. First, $K_{0}=\partial F_{0}$ is the pretzel link $P\left(2,2 x_{1}, 2 y_{1}\right)$. Then, using (15), we can show:

(1) A Seifert matrix $M_{0}$ of $K_{0}$ is singular if and only if $x_{1}=y_{1}=-2$. Since $F$ is of minimal genus, it follows that $K$ is not fibred no matter the value of $\Delta_{K}(0)$.

(2) $\left|\operatorname{det} M_{0}\right|=1$ if and only if

(a) $x_{1}=-1$ and $y_{1}$ is arbitrary, or

(b) $x_{1}$ is arbitrary and $y_{1}=-1$, or

(c) $\left(x_{1}, y_{1}\right)=(-2,-3)$ or $(-3,-2)$.

For cases (a) and (b), $K_{0}$ is fibred and, since $\Delta_{K}(t)$ is monic, $\left|x_{i}\right|=1$ for $i \geq 2$ and $\left|y_{j}\right|=1$ for $j \geq 2$. Therefore, $K$ is fibred by Theorem 3.2(II-i). For case (c), although $\Delta_{K_{0}}(t)$ is monic, $K_{0}$ is not fibred and hence $K$ is not fibred, but $\Delta_{K}(t)$ 
is monic. If $K=M\left(-\frac{1}{2}, \frac{\beta_{2}}{\alpha_{2}}, \frac{\beta_{3}}{\alpha_{3}} \mid e\right)$, take the mirror image and apply the previous argument.

Thus we have the following conclusion:

Theorem 8.4. Let $K=M\left(\frac{1}{2}, \frac{\beta_{2}}{\alpha_{2}}, \frac{\beta_{3}}{\alpha_{3}} \mid e\right)$ be a Montesinos knot in class (3). Let $\left[2 x_{1}, 2 x_{2}, \ldots, 2 x_{p}\right]$ and $\left[2 y_{1}, 2 y_{2}, \ldots, 2 y_{q}\right]$ be the even continued fractions of $\frac{\beta_{2}}{\alpha_{2}}$ and $\frac{\beta_{3}}{\alpha_{3}}$, respectively. Suppose that $\Delta_{K}(t)$ is monic.

(1) If $e \neq 0$, then $K$ is fibred.

(2) Suppose $e=0$. If $\left(x_{1}, y_{1}\right)$ is none of the pairs $(-2,-2),(-2,-3),(-3,-2)$, then $K$ is fibred.

Examples 8.5. (1) $K_{1}=M\left(\frac{1}{2}, \frac{2}{7}, \frac{2}{7} \mid 1\right)=M\left(-\frac{1}{2}, \frac{2}{7}, \frac{2}{7} \mid 0\right)$ is not fibred, since degree of $\Delta_{K}(t)$ is not equal to $2 g\left(K_{1}\right)$. In fact, $M_{0}$ is singular. Note that $\frac{2}{7}=[4,2]$.

(2) $K_{2}=M\left(\frac{1}{2},-\frac{2}{7},-\frac{2}{11} \mid 0\right)$ is not fibred, but $\Delta_{K}(t)=1-t+t^{2}-t^{3}+t^{4}$ is monic, and $g\left(K_{2}\right)=2$. Note that $-\frac{2}{11}=[-6,-2]$.

\section{Acknowledgements}

Hirasawa thanks D. Gabai for encouragement in completing this paper.

\section{References}

[Burde 1984] G. Burde, "Über das Geschlecht und die Faserbarkeit von Montesinos-Knoten", Abh. Math. Sem. Univ. Hamburg 54 (1984), 199-226. MR 87b:57006 Zbl 0557.57004

[Gabai 1983] D. Gabai, "The Murasugi sum is a natural geometric operation", pp. 131-143 in Lowdimensional topology (San Francisco, 1981), edited by S. J. Lomonaco, Contemp. Math. 20, Amer. Math. Soc., Providence, RI, 1983. MR 85d:57003 Zbl 0524.57004

[Gabai 1985] D. Gabai, "The Murasugi sum is a natural geometric operation, II", pp. 93-100 in Combinatorial methods in topology and algebraic geometry (Rochester, NY, 1982), edited by J. R. Harper and R. Mandelbaum, Contemp. Math. 44, Amer. Math. Soc., Providence, RI, 1985. MR 87f:57003 Zbl 0584.57003

[Gabai 1986a] D. Gabai, "Detecting fibred links in $S^{3}$,, Comment. Math. Helv. 61:4 (1986), 519555. MR 88c:57009 Zbl 0621.57003

[Gabai 1986b] D. Gabai, Genera of the arborescent links, Mem. Amer. Math. Soc. 339, Amer. Math. Soc., Providence, 1986. MR 87h:57010 Zbl 0585.57003

[Kanenobu 1979] T. Kanenobu, “The augmentation subgroup of a pretzel link”, Math. Sem. Notes Kobe Univ. 7:2 (1979), 363-384. MR 81a:57008 Zbl 0456.57002

[Klimenko and Sakuma 1998] E. Klimenko and M. Sakuma, "Two-generator discrete subgroups of $\operatorname{Isom}\left(\mathbf{H}^{2}\right)$ containing orientation-reversing elements", Geom. Dedicata 72:3 (1998), 247-282. MR 2000a:20111 Zbl 0928.20040

[Morimoto et al. 1996] K. Morimoto, M. Sakuma, and Y. Yokota, "Identifying tunnel number one knots”, J. Math. Soc. Japan 48:4 (1996), 667-688. MR 97g:57010 Zbl 0869.57008 
Received October 19, 2004. Revised July 31, 2005.

MiKAMI HiRAS AWA

DEPARTMENT OF MATHEMATICS

GAKUSHUIN UNIVERSITY

TOKYO 171-8588

JAPAN

hirasawa@math.gakushuin.ac.jp

Kunio Murasugi

DEPARTMENT OF MATHEMATICS

UNIVERSITY OF TORONTO

TORONTO, ON, M5S 2E4

CANADA

murasugi@math.toronto.edu 